\title{
$1 \quad$ Modeling population size independent tissue epigenomes by 2 ChIL-seq with single-thin sections
}

3 Kazumitsu Maehara ${ }^{1}$, Kosuke Tomimatsu' ${ }^{1}$, Akihito Harada ${ }^{1}$, Kaori Tanaka ${ }^{1}$, Shoko Sato ${ }^{2}$, Seiji

4 Okada $^{3}$, Tetsuya Handa ${ }^{4}$, Hitoshi Kurumizaka ${ }^{2}$, Hiroshi Kimura ${ }^{4}$, and Yasuyuki Ohkawa ${ }^{1^{\star}}$

$5 \quad{ }^{1}$ Division of Transcriptomics, Medical Institute of Bioregulation, Kyushu University, 3-1-1

6 Maidashi, Higashi-ku, Fukuoka 812-0054, Japan.

$7 \quad{ }^{2}$ Institute for Quantitative Biosciences, The University of Tokyo, 1-1-1 Yayoi, Bunkyo-ku, Tokyo

8 113-0032, Japan.

$9 \quad{ }^{3}$ Division of Pathophysiology, Medical Institute of Bioregulation, Kyushu University, 3-1-1

10 Maidashi, Higashi-ku, Fukuoka 812-0054, Japan.

$11{ }^{4}$ Cell Biology Center, Institute of Innovative Research, Tokyo Institute of Technology, 4259

12 Nagatsuta, Midori-ku, Yokohama 226-8503, Japan

13 "Corresponding author: Yasuyuki Ohkawa

14 Email: yohkawa@bioreg.kyushu-u.ac.jp 


\section{Abstract}

17 Recent advances in omics studies have enabled analysis at the single-cell level; however,

18 methods for analyzing the whole cell of large organs and tissues remain challenging. Here, we

19 developed a method named tsChIL to understand the diverse cellular dynamics at the tissue

20 level using high-depth epigenomic data. tsChIL allowed the analysis of a single tissue section

21 and could reproducibly acquire epigenomic profiles from several types of tissues, based on the

22 distribution of target epigenomic states, tissue morphology, and number of cells. The proposed

23 method enabled the independent evaluation of changes in cell populations and gene activation

24 of cells in regenerating skeletal muscle tissues, using a statistical model of RNA polymerase II

25 distribution on gene loci. Thus, the integrative analysis by tsChIL can elucidate in vivo cell-type

26 dynamics of tissues.

\section{Introduction}

28 Tissues are terminally differentiated cells formed from stem cells, followed by cell-type

29 conversion and functional arrangement of cell types to the specified spatial localization.

30 Presently, the composition of the cells playing different functions and the mechanism by which

31 each type is formed have been elucidated. This allowed us to understand the biological function

32 of each tissue and the pathogenesis and developmental failure of diseases. Tissue composition

33 can be determined using known cell-type markers. Immunostaining for cell surface antigens and

34 other cell-type markers enables visual examination that determines the number and localization

35 of cells and tissue morphology. Determining the cell types and the size of the population (i.e.

36 number of cells) in tissues can be done using scRNA-seq, which is based on the transcriptomic

37 differences of individual cells ${ }^{1}$. Unsupervised clustering ${ }^{2}$ of the gene-expression profiles allowed

38 the identification of the cell types, including those previously unknown.

40 Epigenomic analysis is widely performed at the tissue level, such as in the Encyclopedia of DNA

41 Elements (ENCODE), the National Institutes of Health Roadmap Epigenomics Project, and

42 International Human Epigenome Consortium (IHEC) projects that utilize ChIP-seq for bulk-level

43 tissues. The comprehensive identification of functional elements in genomes, such as promoters,

44 enhancers, and the binding sites of transcription factors and their regulatory relationships that

45 characterize the tissues has been achieved ${ }^{3-5}$. However, in epigenomic analysis at tissue-level,

46 imbalance sampling cannot be avoided because tissues are mixtures of diverse cell types.

47 Particularly, when the number of target cells (e.g. stem cells) is limited, they are overwhelmed by 48 the information on the other majority cells. Furthermore, in ChIP-seq, the genome coverage per 49 cell is limited ${ }^{6}$, i.e. information on minority cells in the bulk tissue is lost. Therefore, it is necessary 50 to collect a large amount of target cells that meet the requirements of ChIP-seq. As such, after 
51 defining the target cell types and markers, the sectioning of narrower area, dissection, or 52 cell-sorting is utilized. Recently, new methods for analyzing a small number of cells with higher 53 genome coverage at the single-cell level, including our ChIL and others, have been developed ${ }^{7-}$ $54 \quad{ }^{14}$. In addition, isolating cells causes potential effects to cells owing to the physical separation of 55 the tissues. Several tissue analysis methods that do not involve enzymatic digestion, have also 56 been proposed ${ }^{15-20}$. However, obtaining epigenomic information from a limited number of cells 57 using ChIP-seq based technology remains a challenge. To understand the formation of all cell 58 types, the use of whole-tissue analysis using single-cell technology is ideal; however, it is very 59 costly.

Hence, several transcriptomic analysis approaches that combine the advantages of bulk RNA-seq and scRNA-seq, which can analyze and identify numerous cells at once, have been proposed. For example, the changes in the gene expression of cell types in bulk RNA-seq profiles during embryogenesis have been interpreted using single cell RNA-seq collected separately ${ }^{21}$. The estimation of the cell-type composition of bulk tissue RNA-seq based on single cell RNA-seq has also been reported ${ }^{22}$. Because data from different platforms complement each other, a data integration method has also been proposed, particularly the embedding of single cell RNA-seq into seqFISH $+{ }^{23}$ data to virtually reconstruct whole gene expression data using spatial information ${ }^{24,25}$. In addition, a computational approach for epigenomic analysis to decompose DNA methylation states into cell types has been suggested ${ }^{26}$. However, to date, a universal solution for the cell-type decomposition of tissue epigenomes has not yet been established.

Here, we propose a framework that integrates ChIL into the tissue-slice analysis and uses single, very small, and thin tissue sections for ChIL-Seq (herein, tsChIL). Our obtained bulk tissue epigenome data showed dynamic changes in both the number and cell type, and computational modeling was thus required. We first optimized ChIL for highly sensitive genome-wide analysis using a single thin section, as well as tissue visualization using immunostaining. The ChIL is proposed to enable epigenomic analysis at the single-cell level, and the acquired thin-section tsChIL data are expected to be the sum of the high-depth single-cell epigenomes. Using three different types of tissues, we confirmed the adequate sensitivity, specificity, and reproducibility of tsChIL in identifying enhancers, transcription factors, and transcriptionally activated genes in whole tissues. Thus, we built a statistical model that evaluates the changes in the distribution of RNA Polymerase II at the gene loci and provides a robust, cell-type resolution transcriptional regulatory analysis for large changes in population size. 


\section{Results}

88

\section{Thin-section ChIL-seq enabled spatial epigenomics with single tissue section}

Various cell types exist in tissues, each of which exhibits a unique localization pattern. The transcriptomic and epigenomic pattern of these cells may be affected by the enzymatic isolation process. Therefore, we focused on the use of tissue sections that are free from enzymatic treatment biases for epigenomic analysis and developed an experimental procedure using a single, very small, and thin tissue sections. We then optimized the ChIL for tissue (Fig. 1A), based on our previously reported sc-epigenomic analysis tools ${ }^{7,8}$.

Since the reports on analysis using microtissue sections are limited, and all of them require multiple tissue slices to obtain the required cell number ${ }^{15-20}$ (Table S1). We therefore focused on preparing frozen, unfixed tissue sections to equalize the fixation conditions. On plates, unfixed tissue thin sections are fixed with paraformaldehyde then permeabilized, followed by blocking. Immunostaining is then performed by reacting with primary antibodies against the target molecules on chromatin. Then, a fluorescent-labeled ChIL-probe attached with secondary antibodies was used to obtain the tissue localization of the target by imaging at the subcellular level. Subsequently, Tn5 transposase inserts an artificial sequence containing a T7 promoter into the genomic region near the target. In vitro transcription of the genome sequence near the target protein, starting from the T7 promoter, was performed, and the reverse-transcribed DNA was sequenced using a next-generation sequencer. Compared with conventional epigenomic analysis methods for FFPE and fresh frozen tissue slices, this method enabled uniform fixation conditions for the analysis of micro-thin slices. Therefore, using the highly efficient ChIL method, we attempted to analyze tissues with an input size of $3 \mathrm{~mm} \times 3 \mathrm{~mm} \times 10 \mu \mathrm{m}$. Thus, we designed tsChIL as a high-precision method for analyzing the epigenetic information of a group of cells on a tissue section of the target, following the spatial distribution of the specific epigenetic status.

114 To evaluate the designed tsChIL experimental procedure, the levels of the enhancer marker of 115 histone modification H3K27ac and the recruitment of RNA Polymerase II (RNAPII), an indicator 116 of transcription, were detected in three different tissues: liver, heart (left ventricle), and testis.

117 Most of the cells were hepatocytes, comprising $70-80 \%$ of the liver. The H3K27ac signal 118 visualized by the ChIL-probe was uniformly distributed across cells on the sections. 119 Subcellularly, the co-localization of H3K27ac and RNAPII in euchromatin regions 120 (Hoechst-negative) was observed (Fig. 1B). In the testis, which consists of cells at multiple differentiation stages, the RNAPII signal was strongly distributed and localized in cells with high transcriptional activity, especially near the outer periphery of the seminiferous tubule ${ }^{27}$ (Fig. 1C), 
123 a region where cells in the early stages of sperm differentiation are located (Fig. 1C). Meanwhile, 124 the heart was co-stained using laminin and the ChIL probe to distinguish the cell boundary 125 regions and visualize the basement membrane (Fig. 1D). S5P signal showed a localization to 126 the low Hoechst-dense region of the cell nucleus in which transcription may active, suggesting 127 that immunostaining with ChIL probe was a valid histological staining method at the subcellular level (Fig. 1B-D, Fig. S1).

To validate the feasibility of tsChIL for sensitive and accurate epigenomic analysis, we performed tsChIL-seq using a single thin section containing 1,000-10,000 cells (Table 1), which was generally assumed as a low number of cells in culture ${ }^{7,8}$. The number of cells used was less than that of conventional epigenomic methods used especially for tissue analysis (Table S1). Furthermore, the genome-wide analysis was performed by ChIL reaction on single sections of the sections that showed in Figure 1B-D. In the representative visualized epigenomic data in liver (Fig. 1E), the accumulation of H3K27ac and RNAPII at the Alb locus, a hepatocyte marker, was observed. The former showed an activated upstream enhancer region, whereas the latter was highly transcriptional activity at the locus. The transcription of Alb was also confirmed using RNA-seq with different serial slices. These results indicate that tsChIL enables the simultaneous acquisition of both the tissue distribution of the epigenomic status and the genome-wide epigenomic data using a single tissue section containing a small number of cells $\left(10^{3}\right.$ to $10^{4}$ cells in $10 \mathrm{~mm}^{2}$ area).

Next, to evaluate the genome-wide distribution of the signals obtained using the tSChIL procedure proposed above, we examined the specificity of the signal localization among different tissues and antibodies and the reproducibility of signal localization of the same tissue and antibody. First, to estimate the appropriate number of reads for ChIL-seq with tissues, we obtained $480 \mathrm{M}$ reads from RNAPII ChIL-seq in muscle tissue and evaluated the library complexity $^{28}$ (i.e. the prediction curve of usable reads). As seen in Figure 1F, the number of total usable reads was starting to move away from the black line at approximately $10^{7}$, indicating a decreasing percentage of usable reads. Therefore, we determined that approximately $10^{7}$ reads is a good cost-balanced number of the required reads in the case wherein the number of cells per section is $<10^{4}$. To obtain a ChIL signal with sufficiently high signal-to-noise ratio, we acquired an average of approximately $14 \mathrm{M}$ reads (Table S2), which is comparable to the number of reads in the ENCODE tissue ChIP-seq $(10 \mathrm{M}-20 \mathrm{M})^{3}$. 
H3K27ac and RNAPII S5P antibodies, signals were concentrated around the coding regions (promoters and gene body) compared with the no-antibody (herein, No Ab; without primary antibody) controls (53\%-59\% and $41 \%-48 \%$, respectively). The results showed that the genomic sequences were selectively extracted from the transcriptionally activated regions of the genome. In Figure $\mathbf{1 H}$, we describe the correlation matrix of the signal levels on the whole genome to confirm the high reproducibility of the replicates. The dendrogram shows the hierarchical structure of the highest correlation among the replicates (Liver-H3K2ac: 0.90, Liver-Polll: 0.90, Heart-H3K27ac: 0.87, Heart-Polll: 0.92, Testis-H3K27ac: 0.91, Testis-Polll: 0.94 in average of triplicates), and the correlation within the same tissue (e.g., Liver-Polll vs. Liver-K27ac: 0.87; Heart-H3K27ac vs. Heart-Polll: 0.88 ; and Testis-H3K27ac vs. Testis-Polll: 0.88 ; the list of all correlation coefficients are summarized in Table S3). These results suggest that tsChIL-seq can capture the epigenomic differences between different tissues and is technically reproducible.

Identification of regulatory factors in the formation of tissue-specific enhancers

173 We next assessed the ability of tsChIL for low-input epigenomic analysis of tissues. First, we performed tsChIL using thinly sectioned tissues from the liver, heart, and testis, and the identified enhancers were compared by matching references ${ }^{3}$ (Fig. 2A). According to the odds ratio (i.e., specificity, the detailed definition is described in Method), each H3K27ac ChIL-seq signal preferentially captured the corresponding tissues-specific enhancer (Liver: 33.5, Heart: 27.1, Testis: 4.1; The other odd ratios are listed in Table S4). Therefore, we successfully detected tissue-specific enhancers using tsChIL with lower input compared to the previous reports that utilized $500 \mu \mathrm{g}$ chromatin equivalent to $10^{7}-10^{8}$ cells.

Next, we examined the enrichment of the H3K27ac signal on representative tissue-specific enhancers, including the liver, heart, and testis. We focused on the enhancer region of Rxra genes $^{29}$ specifically expressed in liver tissues, Gnat3 cardiac muscle-specific gene retinoic acid receptor, and Eps8 expressed in the blood-testis barrier $(\mathrm{BTB})^{30}$. H3K27ac signal enrichments on each tissue-specific enhancer were observed on the IGV screen shot (Fig. 2B). In contrast, all Actb-expressing tissues showed the ubiquitous enrichment of H3K27ac.

We further evaluated the enrichment of the regulatory sequence in extracted enhancers using tsChIL based on the enrichment of the TF-binding motif (only the top scoring motifs are shown in

191 Fig. 2C; all others are in Table S5). The enrichment of known liver-specific TF-binding motifs,

192 Rxra, Hnf4a, Nr2f6, and others were observed in the H3K27ac tsChIL-seq data obtained from the liver. This data is consistent with the liver-specific regulatory sequences registered as open chromatin regions detected using ATAC-seq with mouse liver tissues in the database ${ }^{31}$. 
195 Meanwhile, the H3K27ac signal obtained from the heart showed relatively higher enrichment at 196 Klf12 than others; Sox5 and androgen receptor (AR) binding motifs were enriched in the 197 testis-H3K27ac signal, which was consistent with previous studies reporting that AR binds to the 198 androgen responsible element (ARE) on regulatory sequences with histone acetyltransferase to 199 regulate gene expression ${ }^{32,33}$. These data support that H3K27ac tsChIL can identify cis-regulatory elements following the extraction of tissue-specific enhancers.

202 Because the enrichment of the tsChIL signal should reflect the quantitative H3K27ac levels as demonstrated by the identification of super enhancers (SEs) using ChIP-seq, we next quantitatively determined the H3K27ac level based on the read counts. Then, SE formation upon TF binding on the extracted cis-regulatory elements was evaluated. First, we listed the highly enriched regions of the H3K27ac tsChIL signal as $\mathrm{SE}^{34,35}$ from each liver, heart, and testis data set. The labeled genes in Figure 2D are representative protein-coding genes near the identified top ranked SEs, which have the highest read counts in peaks (see Fig. S2 for all replicates). In the liver, known hepatocyte marker genes, Alb, and albumin family, Gc are also detected in motif-enrichment analysis performed in Figure $\mathbf{2 C}$. In addition, the core transcription factor $211 \mathrm{Hnf}_{4} \mathrm{a}^{36}$, which activates the genes by itself, was included in the top rank (1.6 to $3.5 \%$ ). 212 Furthermore, the SEs featuring each tissue were identified. In the heart (left ventricle), Ablim1 213 expressed in the left ventricle and involved in left-right axis formation ${ }^{37}$, was detected, whereas 214 in the testis, SEs were identified in the vicinity of Crem, which is involved in spermatogenesis ${ }^{38}$.

216 Finally, to validate the function of the SEs identified in the liver using this method, we performed 217 tsChIL targeting Hnf4a, which showed a high specificity score (deviation-Z) in liver SEs. Hnf4a is 218 known to be an important nuclear receptor during hepatocyte differentiation ${ }^{39}$, and has been 219 shown to contribute to SE formation as a core transcription factor, along with $\mathrm{RXR \alpha ^{29 }}$. 220 Immunostaining with the ChIL Probe showed that the HNF4 $\alpha$ was distributed throughout most 221 cells in the liver tissue and detected in the open chromatin region of the nucleus in each cell (Fig. 222 2E). A pronounced accumulation of $\mathrm{Hnf} 4 \alpha$ signals in the SEs in the region was observed (Fig. $223 \mathbf{2 F}$, see Fig. S3 for the motif enrichment analysis on Hnf4a peaks). We next evaluated the 224 selective binding of Hnf4a to the genes in the liver SEs (Fig. 2G; Fig. S4 for the replicates). 225 Using the gene sets of SEs and TEs neighboring genes obtained in Figure 2D, gene sets enrichment analysis (GSEA) ${ }^{40}$ demonstrated that the hits of the ChIL-Hnf4a peaks against liver enhancers scored as high as 0.72 in the enrichment score (Fig. 2G, top). Particularly, Hnf4a was 228 bound to $76.4-78.8 \%$ of the SEs (Fig. 2G bottom). In contrast, in the negative controls of the heart- and testis-specific SEs, the number of SEs bound by Hnf4a was approximately 0.5 in the 
230 enrichment score and the percentage of Hnf4a bound to the heart- and testis-specific SEs was at a random chance level (24.4-34.2\%).

233 In summary, the data from tsChIL-H3K27ac demonstrated that the regulatory candidate 234 transcription factor Hnf4a obtained from the cis-element refinement selectively binds to the 235 liver-specific SE region of the Hnf4a locus. Hnf4a could be validated to provide positive feedback 236 that binds to the SE region of its own Hnf4a locus. Our data indicated that tsChIL is useful for the 237 regulatory analysis of enhancers, including transcription factors and SEs, using low number of 238 cells.

\section{tsChIL-RNAPII peaks detected the majority of active genes in tissue}

241 Transcriptome information is obtained by evaluating the binding position of RNAPII using 242 epigenomic analysis. Here, we detected the active genes based on the binding of RNAPII on the 243 genome using tsChIL. In Figure 3A, we plotted the cumulative number of consumed reads of the 244 detected genes in RNA-seq and RNAPII tsChIL in the order of their read counts. Due to the wide 245 dynamic range of RNA-seq data, high copy-number mitochondrial-derived RNAs (e.g., 246 mitochondrial ribosomal RNAs) and highly expressed genes that characterize each tissue (Alb in 247 liver, Myh6 in the heart, Prm1 in testes), consumed $80 \%$ reads on a small number of highly 248 expressed genes (whose expression can be confirmed; Liver 5\%, Heart 1\%, Testis 11\%). The 249 identification of weakly expressed genes and rare populations in bulk tissue RNA-seq is 250 generally hard to obtain because the top $10 \%$ genes spends $80 \%$ of its reads in even at the 251 single-cell level ${ }^{41,42}$.

253 In contrast, ChIL-RNAPII did not exhibit an exponential increase in the number of consumed 254 reads required to detect gene expression from RNA-seq. It also efficiently detected more genes 255 as the number of reads increased. The dynamic range of RNA-seq depends on the product of 256 the cell number and the concentration of RNA in each gene, whereas that of the RNAPII signals, 257 in essence, depends on the product of the presence or absence of gene expression $(0,1$, or 2$)$ 258 and the cell number. The results are consistent with the fact that highly and ubiquitously 259 expressed genes occupy a high number of reads in the RNA-seq data. The result suggested that 260 fewer reads are required for gene expression profiling using tsChIL RNAP2 than RNA-seq.

262 Thus, the genes were divided into five groups based on their expression levels from RNA-seq, 263 and the correlation of each tsChIL RNAPII signal with their expression levels was examined (Fig. 264 3B). In the high-expression group in all tissues, the intensity of the RNAPII signal in the TSS was 265 highly correlated with its expression level. In the $75^{\text {th }}-100^{\text {th }}$ percentile group, a high accumulation 
of RNAPII in the gene body region was also detected, suggesting a movement of RNAPII to the locus upon transcriptional activation. Here, we showed that tsChIL-RNAPII demonstrated a preference for capturing highly expressed genes in tissues. Subsequently, we assessed the overlap between RNA-seq-confirmed genes (TPM >0) and tsChIL-RNAPII peaks. tsChIL peaks captured approximately $30 \%$ (Testis slightly lower, approximately $20 \%$ ) of the active genes (TPM $>0$ ), whereas false positives were almost absent (Fig. 3C). In addition, tsChIL peaks stably

272 detected approximately $40-50 \%$ of the genes expressed in RNA-seq, independent of the TPM

273 threshold for defining the expressed genes in RNA-seq (Fig. S5). These results suggest that the

274 peak region is likely to capture genes with high expression because the region with high signal 275 counts was judged to be the peak region ${ }^{43}$. In all tissues, the expression levels of the genes in 276 Common were higher than those in RNA-seq group as expected (Fig. 3D).

278 Figure 3E shows an IGV screenshot of the tsChIL RNAPII. The accumulation was detected at 279 the Trf (transferrin) locus in the liver, Myh6 (cardiac myosin) in the heart, and Meig1 (a 280 meiosis-expressed gene) in the testes. These are considered representatives of genes 281 specifically expressed in each tissue. At the Actb locus, a house-keeping gene, the RNAPII signal was accumulated in all tissues, indicating active transcription. In these highly transcriptionally active genes, a wide distribution of RNAPII signals was detected on the gene body, suggesting that the RNAPII binding distribution patterns would enable an in-depth profiling of the transcriptional programs in tissues.

Modeling RNAPII traveling reveals transcriptional dynamics in the rapid change of cell population in skeletal muscle regeneration

289 We demonstrated that enhancers and transcriptional activity states can be detected with high sensitivity, specificity, and reproducibility at the whole-tissue level by the optimized ChIL for tissues. Then, tsChIL-RNAPII data in Figure 3 suggested that, in addition to amount of the signal at the gene loci, evaluation of the distribution or its elongation across the entire locus would improve the analysis of the transcriptional activation in various cells in tissue. We thus conceived a concept the statistical modeling of tsChIL-RNAPII data for the epigenomic analysis of heterogeneous tissues.

We used skeletal muscle regeneration as a model case, wherein numerous cell types dynamically change their composition, particularly that of the mouse tibialis anterior (TA) muscle after cardiotoxin (CTX)-induced injury. During regeneration, migrating immune cells are dominate the tissue 2 to 3 days after muscle injury ${ }^{44}$. During this time, the activation of satellite 
302 fibers observed on day 14. We thus established a model to analyze the gene expression

303 dynamics in each cell type from day 0 (pre-injured period) and until day 14. tsChIL obtained data

304 from five biological replicates using the tissue sections of TA muscles at five time points on days

$3050,3,5,7$, and 14 after the CTX-induced muscle injury. As shown in Figure 4A (Fig. S6A for the

306 entire time-course), the basal lamina separating the muscle fibers observed on day 0 was

307 destroyed post-injury. The destruction of the cells on the third day can be seen in the image of

308 laminin co-stained with the ChIL probe. Furthermore, the fluorescence image of the ChIL probe

309 suggests the presence of multiple cell types, such as the activated muscle satellite cells, muscle

310 progenitor cells that have started to differentiate, and migrating immune cells associated with the

311 inflammatory response. On day 14, the structure of the muscle fibers possessing central nuclei

312 were observed, thus indicating regenerated muscles.

313

314 First, we visualized the distribution of the RNAPII signal by IGV for representative genes in 315 skeletal muscle and immune cells. Changes in RNAPII distribution are observed at the locus for 316 Acta1 (which is highly expressed in skeletal muscle) and Cd68 (a surface marker of 317 macrophages) (Fig. 4B). The Cd68 locus showed an overall increase in the RNAPII signal from 318 day 0 to day 3, whereas Acta1 showed an overall decrease. These results indicate the rapid 319 increase in immune cells and the decrease in skeletal muscle cells during the early stages of 320 injury (days 2-3) as shown in Figure 4C. In Acta1, however, the RNAPIl signal is more 321 concentrated near the transcriptional end site (TES) than the transcriptional start site (TSS). We 322 thus hypothesized that the shape of the RNAPII distribution contains information on both the 323 population size of cells and the regulatory state of a gene known as the pause/release of the 324 RNAPI ${ }^{45,46}$. Therefore, we established a model for two cases (or their combination) as shown in 325 Figure 4D: one in which a specific gene of resident cells is activated by the induction of muscle 326 regeneration (i), and the other in which the height of the already activated RNAPII signal 327 increases due to an increase in the number of cells (e.g., migrated immune cells from outside the 328 tissue) (ii). The traveling ratio (TR) is often used to evaluate the degree of RNAPII pause/release, 329 as in Bartman et al. ${ }^{47}$, providing a brief description of the geometry of the distribution of the 330 RNAPII in the gene loci in terms of the ratio of the signal levels between TSS and TES. 331 Furthermore, we modeled the estimation of TR as a form of Poisson regression with an offset 332 term (see details in Methods). For each locus, the signal level (count per million [CPM]) of 333 RNAPII at the TSS is $\exp \left(\beta_{0}\right)$, and that of TES is $\exp \left(\beta_{1}\right)$ times the TSS level $\exp \left(\beta_{0}\right)$, i.e., $334 \exp \left(\beta_{0}+\beta_{1}\right)$. The statistical model allows us to evaluate the confidence intervals for TR and 335 perform statistical tests for changes in varying conditions and time points. 
337 Figure 4E shows the estimated values of the mean RNAPII levels at TSS and TR, along with the 338 confidence intervals. We then compared the tissue-wide expression levels of the corresponding 339 genes (Fig. 4F). Surprisingly, the tissues-wide expression of Acta1 and Cd68 were synchronized 340 with the pattern of the RNAPII TSS-level, whereas the transcription factor myogenin (Myog) 341 expressed in muscle progenitor cells at the differentiation stage has a synchronized pattern to 342 TR. These results suggest that the tissue bulk RNA-seq is a combination of the cell number and 343 the changes in the amount of gene expression.

345 Therefore, to distinguish the transcriptional activation indicated by the TR, and the population 346 size indicated by the TSS-level as inferred in Figure 4E-F, we analyzed the changes in the 347 TSS-levels and TR at day 3 (Fig. 4G and Fig. S6C). Each set of genes was associated with each 348 'single' cell-type, the definition of which is based on the scRNA-seq analysis of injured muscle by 349 De Micheli et al. ${ }^{48}$. The population size of the cells that express the skeletal muscle related 350 genes (Fig. 4G, right) were decreased after injury, whereas the changes in TR revealed the 351 active transcription of the genes. Meanwhile, in the group of genes associated with immune 352 cells, TSS-level was increased while TR was less altered (Fig. 4G, left), which can be interpreted 353 as an increase in the population of cells already possessing active gene loci (i.e. migration). This 354 interpretation is consistent with the dynamic population changes in muscle regeneration clearly 355 revealed by recent studies using scRNA-seq ${ }^{48-50}$. In summary, the statistical model of tsChIL 356 RNAPII allowed us to evaluate the transcriptional activity of genes associated with specific cell types, independent of increased population of immune cells and decreased skeletal muscle cells

360 Next, we identified the uncharacterized dynamics in muscle regeneration from day $0-14$ using 361 the other cell-type markers defined by De Micheli et al. ${ }^{48}$. First, we selected 66 genes among the 362 markers that changed the TR $(F D R<0.1)$ at any time point compared with day 0 . The changes 363 in the TR and TSS level of these genes are shown as a heatmap (Fig. $\mathbf{4 H}$ ) to visualize the trends 364 in the transcriptional activation of each gene, as well as the increase or decrease in the number 365 of cells that harbor the activated genes. From the $\log _{2}$ TSS, which indicates the cell number, we 366 confirmed that mature skeletal muscles (SKMs) decreased once after injury (white to blue); 367 however, most genes were activated at day 3 and returned to the original population size (white) 368 at day 14. Many of the cell types, such as mesenchymal progenitors/SMCs, myeloid progenitors, 369 and resident macrophages/APCs, transiently increased in number after injury but returned to 370 their pre-injured levels on day 14, indicating association with inflammatory responses (Ada2, 371 Rgs2, Coro1a, Lyz2, C1qa) ${ }^{50,51}$. Meanwhile, Myl1, a gene that was transiently increased after 
372 injury, Tnnc2, and Acta1, showed the same TR pattern, suggesting that these genes also

373 function in regeneration and not only in muscle fiber formation ${ }^{52}$.

375 Next, we describe the muscle regeneration process by classifying gene groups according to the

376 pattern of TR changes over time. The clusters C1-5 were assigned according to their peaks

377 (highest point) of TR in the time-course of regeneration, the tissue-wide dynamics were

378 appeared in Figure $\mathbf{4 I}$, suggesting transcriptional regulation in muscle regeneration. The C1

379 exhibit the highest TR at day 0 , and thus indicates a down regulated biological process after the

380 injury. The proliferation of the immune cell was repressed, and the major participants are the

381 resident macrophages and APCs and myeloid progenitors. The C2 which has peak at day 3 ,

382 districted the activation of myogenesis mainly orchestrated by MuSC, muscle progenitors and

383 also by neural cells, which is consistent with previous reports ${ }^{53}$. The $\mathrm{C} 3$, which has peak day 5 ,

384 does not show strong enrichment. The C4 contained muscle contraction, ion transport and action

385 potential related GO terms, which suggests the regenerated muscle was formed at day 7 . The

386 C5 (day 14) showed the activation of angiogenesis in the late stage of regeneration ${ }^{54}$. Here, the

387 statistical modeling that combined RNAPII-mediated transcriptional elongation and population

388 size changes achieved by our tsChIL provides a strategy for understanding the process of

389 muscle regeneration that is organized by diverse cell types in tissue.

390

\section{Discussion}

392 Here, we established a high-precision method for tissue epigenomic analysis using a single, thin 393 section samples. We focused on the tsChIL data of RNAPII and established a statistical model to 394 identify the changes in both population size and transcriptional regulation in the various cell

395 types. In this analysis, we utilized single-cell analysis transcriptomic data as a reference of

396 cell-type annotation. The efficient combination of existing single-cell analysis data and bulk but

397 high-depth tsChIL data may lead to future approaches to analyze large numbers of individuals at

398 the whole-cell level.

400 We demonstrated that the transcriptional regulation of each cell type can be analyzed

401 independently, even in situations with large-scale variations in tissue cell-type composition, as in

402 the case of muscle regeneration. tsChIL by itself can also provide a qualitative assessment of the

403 changes in cell population size. Although we did not identify the cell types in the tissues nor

404 estimated their compositional ratios, our framework that combined scRNA-seq and epigenomic

405 analysis provides solid guidance for future tissue analysis. 
407 The traveling ratio (or pausing index), a concise measure of RNA polymerase II dynamics, which was originally introduced in the ChIP-chip as a measure of the degree of transcriptional

409 elongation ${ }^{45,46}$; and used in GRO-seq ${ }^{55}$ and ChIP-seq ${ }^{56}$. We found that the shape of the

410 distribution of RNAPII at the genomic locus, as revealed by epigenomic analysis, is indeed a

411 useful indicator of the transcriptional activity of a gene, and that the RNA-seq of bulk tissue is the sum of all transcripts of all cells and is always affected by the population size.

414 The statistical modeling of TR provides analogous advantages in the analysis of differentially

415 expressed genes, such as the screening of genes with altered transcriptional states and 416 calculation of confidence intervals for TR. Here, we used a simplified model in which the RNAPII 417 signal at a single locus is the product of the size of the active population and the degree of 418 activity (traveling ratio). Alternatively, a more realistic model with different transcriptional 419 activities for different cell types and within the same cell type may be possible as proposed in the 420 bulk data decomposition methods ${ }^{22,57,58}$. Despite our simplified assumption, our established 421 model successfully determined transcriptional activities by cell type within a tissue. In addition, 422 tsChIL RNAPII data can be modeled using a simple Poisson distribution rather than a negative 423 binomial distribution, which involves a complex dispersion parameter estimation. Furthermore, 424 the use of CPM normalization with offset terms as a natural way of handling replicates made the 425 model easier to apply, interpret, and use for tissue epigenome profiling.

427 Conventional ChIP-seq has a limited genome coverage of cell owing to the efficiency of 428 immunoprecipitation. In contrast, ChIL-seq, on which tsChIL is based, achieves a higher genome 429 coverage of at least $90 \%$ for histone modifications at the single-cell level. Accordingly, the 430 acquired data was assumed to be a sum of the deeply profiled cells. Thus, we believe that the 431 acquisition of such high-depth epigenome data will continue to be necessary for the modeling 432 compositions of tissues as shown in our framework. These high-depth data are expected to be 433 provided not only by ChIL-seq, but also by other single-cell epigenomic analysis methods; thus, other methods can be integrated to our analysis framework.

436 tsChIL showed great potential to replace ChIP-seq, which has been the standard method of 437 epigenomic analysis for tissues. In this paper, the high reproducibility of tsChIL, both technically 438 and biologically, was demonstrated. Furthermore, tsChIL achieved comparable performance 439 while using fewer cells than ChIP-seq ( 1/10,000 of required cell), and parameters, such as 440 fixation conditions, can be monitored based on the quality of immunostaining images. These 441 advantages can reduce cost. In addition, by combining visualization and genome-wide analysis 442 the spatial characteristic can be profiled and linked with the genome-wide characteristics of 
443 epigenomes as shown in the massive wave of RNAPII in the testis. For more advanced

444 applications, by leveraging the pairing of serial-thin sections of the same mouse, the correlation

445 between spatial and genome-wide patterns of heterologous proteins, such as histone

446 modifications and transcription factors, may be reliably estimated. We believe our proposed

447 method is a useful tool for tissue epigenomic analysis, together with recent scRNA-seq and

448 microscopy-based spatial transcriptomics.

449

450

\section{Materials and Methods}

451 Ethical statement

452 All animal procedures were conducted in accordance with the Guidelines for the Care and Use of

453 Laboratory Animals and were approved by the Institutional Animal Care and Use Committee

454 (IACUC) at Kyushu University.

455

\section{Tissue preparation}

457 Eight-week-old C57BL/6N mice were used as replicates for this study. The liver, left ventricle and 458 testis were prepared from male, and tibialis anterior (TA) muscles were from female mise.

459 Tissues were freshly frozen using isopentane chilled with $\mathrm{LN} 2$ and stored at $-80^{\circ} \mathrm{C}$. Muscle

460 regeneration studies were performed as previously reported, except for the injection of CTX into

461 the TA muscle ${ }^{59}$. Injured and intact TA muscles were sampled from five mice at day 0, 3, 5, 7,

462 and 14 after CTX injury. The day 0 indicates a needle-injured control.

463

\section{Immunohistochemistry}

465 Each tissue cryosection $(10 \mu \mathrm{m})$ was placed on the bottom of 96-well microplate (Ibidi \#89626)

466 and stored at $-80^{\circ} \mathrm{C}$ until use. Each section was fixed with $4 \%$ paraformaldehyde in $0.3 \%$

467 TritonX-100/PBS for 5 min and washed with 0.3\% Triton X-100/PBS. Double blocking was

468 performed using blocking one (Nacalai \#03953) and M.O.M blocking reagent (Vector

469 Laboratories \#BMK-2202) following the manufacturer's protocol. The sections were incubated

470 overnight at $4 \stackrel{\circ}{\circ}$ with primary antibodies diluted in M.O.M. protein concentrate/PBS, followed by

471 incubation with ChIL probe at the same conditions but with the addition of $0.5 \mathrm{M} \mathrm{NaCl}$. Then, the

472 wells were filled with PBS for imaging. The following antibodies were used: rabbit anti-H3K27ac

473 (1:500) (CMA309/9E2H10) ${ }^{60}$, rat anti-RNA polymerase II S5P $(1: 1000)(1 \mathrm{H} 4 \mathrm{~B} 6)^{61}$, and rabbit

474 anti-HNf4a (1:500) (C11F12, Cell Signaling Technology Cat. \#3113), rabbit anti-laminin2a

475 (Sigma \#L-9393).

476

$477 \quad$ tsChIL-seq 
478 tsChIL-seq was performed according to $\mathrm{ChIL}^{7,8}$ with some modifications: longer incubation time 479 was employed for some steps ( $1 \mathrm{~h}$ extended Tn5 binding and $2 \mathrm{~h}$ fill-in step), Thermo T7 RNA 480 polymerase (100 U/well; Toyobo), and 15 cycles of polymerase chain reaction (PCR)

481 amplification. Column purification (Zymo \#D4013) and ×0.5 volume of AMpure beads (Beckman 482 Coulter) selection were performed to obtain 200 to 500 bp average of the library. The single-end 483 libraries were sequenced using NovaSeq (Illumina). Reads were mapped against the GRCm38 484 reference genome using Bowtie ${ }^{62}$ with the default option. Duplicated reads were discarded 485 using Samtools (rmdup). The uniquely mapped reads were used for further analysis.

486

\section{Quality assessments of tsChIL-seq data}

488 The matrix of read counts on the equally sized $(10 \mathrm{~kb})$ windows on the mouse genome was 489 generated using deepTools ${ }^{63}$ (version. 3.4.1) with the command: multiBamSummary bins -bs 49010000 --ignoreDuplicates. Pearson correlation coefficients were calculated using the 491 log-transformed read count (with +0.5 pseudo-counts). The breakdown of mapped reads at the 492 genomic regions was calculated using HOMER (annotatePeaks.pl). The library complexity was 493 evaluated by Preseq ${ }^{28}$. The theoretical case assumed uniform probabilities of obtaining reads from the mouse genome (i.e., a common expected values of the Poisson distribution).

\section{RNA-seq analysis}

497 Total RNA (10 $\mathrm{ng}$ ) was extracted for library preparation using a SMART-Seq Stranded Kit

498 (Takara) according to the manufacturer's instructions. Libraries were sequenced on Hiseq1500 499 and NovaSeq (Illumina). Gene expression quantification was performed using Salmon ${ }^{64}$ quant 500 with the default option.

503 Peaks of tsChIL-H3K27ac were called using MACS2 ${ }^{65}$ with the option: callpeak --call-summits $504--n o m o d e l$--nolambda -q 0.05. Tissue specificities of the peaks were evaluated using the odds 505 ratio in the known tissue-specific enhancer lists ${ }^{3}$. The odds ratio is defined as $(p /(1-p)) /(q /(1-q))$, 506 where $p$ is the proportion of hits in the target tissue and $q$ is the proportion of hits to the other 507 tissues in the enhancer lists. ChromVAR ${ }^{66}$ analysis was performed using consensus peaks of 508 each tissue. The consensus peaks were constructed by taking the intersection of the peaks of 509 three biological replicates. Typical and super enhancer candidates were called using HOMER ${ }^{67}$ 510 finePeaks with the option: -style super -superSlope -1000 -gsize 3e9. The pre-ranked GSEA ${ }^{40}$ 511 was performed using tag (read) count-ordered enhancer peaks. Then, the peaks were marked 512 by a binary indicator overlapping with tsChIL-Hnf4a peaks (called by MACS2 as described above 513 with the option: $-q 1 e-5)$. 
516 Aggregation plots of the gene expression percentile groups were created using agplus ${ }^{68}$. The

517 gene groups were divided according to the TPM of the bulk RNA-seq analysis of each tissue

518 (liver, heart, and testis).

\section{Statistical modeling of traveling ratio}

521 The read counts of RNAPII tsChIL-seq at the TSS-region ( -750 to $+750 \mathrm{bp})$ and TES-region ( 0 to $+1,500 \mathrm{bp}$ ) at all mouse transcripts were fitted to the following Poisson regression model. For each gene, assume that the read count $y_{i j}$ of the $i$-th replicate at site $j$ (TSS or TES) follows the

524 Poisson distribution, where the mean parameter $\lambda_{i j}$ satisfies the relation: $\lambda_{i j} / M_{i}=\exp \left(\beta_{0}+\beta_{1} s_{i j}\right)$.

525 The offset term $M_{i}$ is the total reads (in millions) of the replicate $i$, and $s_{i j}$ is the indicator variable 526 that the read count $y_{i j}$ is either TSS $\left(s_{i j}=0\right)$ or TES $\left(s_{i j}=1\right)$. Since the offsetting is equivalent to 527 the CPM normalization of the mean count, $\exp \left(\beta_{0}\right)$ and $\exp \left(\beta_{1}\right)$ can be referred to as the mean

528 CPM at TSS and the magnification factor of TES to TSS (i.e., the traveling ratio) of the gene,

529 respectively. The model evaluates variance and can thus estimate the confidence intervals of the 530 traveling ratio by utilizing all replicates ( 5 in our case) that have different total sequenced reads.

531 We assumed that the contrasts $X-Y$ (e.g., fold-changes of TR between day 3 and day 0 ) follow a

532 Gaussian distribution, and the variance was calculated from $V_{X}+V_{Y}$ (variances of $X$ and $Y$ )

533 under the independence assumption of $X$ and $Y$. $p$-values were estimated from the model, and

534 multiple test correction was performed using the Benjamini-Hochberg procedure in the selected 535 genes of interest.

536

\section{Data Availability}

538 The RNA-seq and tsChIL-seq data generated in this study have been deposited in the Gene

539 Expression Omnibus (GEO) database under the accession code: GSE159024. The codes used

540 for the statistical modeling of tsChIL-seq data are available at:

541 https://github.com/kazumits/tissueChlL

543 Author Contributions: K.M., K.To., A.H. and Y.O. conceived and designed the experiments.

544 K.Ta. and A.H. performed the experiments. K.M. and K.Ta. analyzed the data. K.M. performed 545 statistical analysis. S.S., S.O., T.H., H.Ku. and H.Ki. contributed materials and analysis tools. 546 K.M., K.To. and Y.O. wrote the paper. All authors read and approved the final manuscript.

547 Competing Interest: The authors declare no competing financial interests except A.H., T.H., H. 548 Ku., H.Ki. and Y.O. who are involved in a pending patent related to ChIL. 


\section{Acknowledgments}

550 Computations were carried out using the computer resources offered under the category of

551 Intensively Promoted Projects by the Research Institute for Information Technology at Kyushu

552 University. We appreciate the technical assistance from The Research Support Center,

553 Research Center for Human Disease Modeling, Kyushu University Graduate School of Medical

554 Sciences. This work was in part supported by JST PRESTO JPMJPR2026 to K.M.,

555 JPMJPR19K7 to A.H., JST CREST JPMJCR16G1 to Y.O., H.Ku. and H.Ki.; JST ERATO

556 JPMJER1901 to H.Ku.; MEXT/JSPS KAKENHI JP19H04970, JP19H03158 and JP2OH05393 to

557 K.M.; JP18K19432, JP19H03211, JP19H05425 and JP20H05368 to A.H.; JP18H05534 and

558 JP20H00449 to H.Ku.; JP18H04802, JP18H05527, JP19H05244, JP17H03608, JP2OH00456

559 and JP2OH04846 to Y.O.; JP18H05527 and JP17H01417 to H.Ki.; AMED JP20ek0109489h0001

560 to Y.O., AMED BINDS JP19am0101076 and JP20am0101076 to H.Ku.; JP19am0101105 to

561 H.Ki.

562

\section{References}

564 1. Schaum, N. et al. Single-cell transcriptomics of 20 mouse organs creates a Tabula Muris 565 the tabula Muris consortium*. Nature (2018). doi:10.1038/s41586-018-0590-4

566 2. Blondel, V. D., Guillaume, J.-L., Lambiotte, R. \& Lefebvre, E. Fast unfolding of 567 communities in large networks. J. Stat. Mech. Theory Exp. 2008, P10008 (2008).

568 3. Shen, Y. et al. A map of the cis-regulatory sequences in the mouse genome. Nature $569 \quad$ (2012). doi:10.1038/nature11243

570 4. Roadmap Epigenomics Consortium et al. Integrative analysis of 111 reference human $571 \quad$ epigenomes. Nature (2015). doi:10.1038/nature14248

572 5. Stunnenberg, H. G. \& Hirst, M. The International Human Epigenome Consortium: A Blueprint for Scientific Collaboration and Discovery. Cell (2016). doi:10.1016/j.cell.2016.12.002

575 6. Rotem, A. et al. Single-cell ChIP-seq reveals cell subpopulations defined by chromatin $576 \quad$ state. Nat. Biotechnol. (2015). doi:10.1038/nbt.3383

577 7. Harada, A. et al. A chromatin integration labelling method enables epigenomic profiling $578 \quad$ with lower input. Nat. Cell Biol. (2019). doi:10.1038/s41556-018-0248-3

579 8. Handa, T. et al. Chromatin integration labeling for mapping DNA-binding proteins and modifications with low input. Nat. Protoc. doi:10.1038/s41596-020-0375-8 
581 9. Kaya-Okur, H. S. et al. CUT\&Tag for efficient epigenomic profiling of small samples and single cells. doi:10.1101/568915

10. Carter, B. et al. Mapping histone modifications in low cell number and single cells using antibody-guided chromatin tagmentation (ACT-seq). Nat. Commun. (2019). doi:10.1038/s41467-019-11559-1

11. Wang, Q. et al. CoBATCH for High-Throughput Single-Cell Epigenomic Profiling. Mol.

12. Skene, P. J., Henikoff, J. G. \& Henikoff, S. Targeted in situ genome-wide profiling with high efficiency for low cell numbers. Nat. Protoc. (2018). doi:10.1038/nprot.2018.015

13. Ku, W. L. et al. Single-cell chromatin immunocleavage sequencing (scChIC-seq) to profile histone modification. Nat. Methods (2019). doi:10.1038/s41592-019-0361-7

14. Ai, S. et al. Profiling chromatin states using single-cell itChIP-seq. Nat. Cell Biol. (2019). doi:10.1038/s41556-019-0383-5

15. Fanelli, M. et al. Pathology tissue-chromatin immunoprecipitation, coupled with high-throughput sequencing, allows the epigenetic profiling of patient samples. Proc. Natl. Acad. Sci. U. S. A. (2010). doi:10.1073/pnas.1007647107

16. Fanelli, M., Amatori, S., Barozzi, I. \& Minucci, S. Chromatin immunoprecipitation and high-throughput sequencing from paraffin-embedded pathology tissue. Nat. Protoc. (2011). doi:10.1038/nprot.2011.406

17. Cejas, P. et al. Chromatin immunoprecipitation from fixed clinical tissues reveals tumor-specific enhancer profiles. Nat. Med. (2016). doi:10.1038/nm.4085

18. Amatori, S. et al. Epigenomic profiling of archived FFPE tissues by enhanced PAT-ChIP (EPAT-ChIP) technology. Clin. Epigenetics (2018). doi:10.1186/s13148-018-0576-y

19. Zhong, J. et al. Enhanced and controlled chromatin extraction from FFPE tissues and the application to ChIP-seq. BMC Genomics (2019). doi:10.1186/s12864-019-5639-8 resolution. / Nat. / 583, (2020). 
611 22. Newman, A. M. et al. Determining cell type abundance and expression from bulk tissues with digital cytometry. doi:10.1038/s41587-019-0114-2

613 23. Eng, C. H. L. et al. Transcriptome-scale super-resolved imaging in tissues by RNA seqFISH+. Nature (2019). doi:10.1038/s41586-019-1049-y

24. Stuart, T. et al. Comprehensive Integration of Single-Cell Data. Cell 177, 1888-1902.e21 (2019).

617 25. Abdelaal, T., Mourragui, S., Mahfouz, A. \& Reinders, M. J. T. SpaGE: Spatial Gene Enhancement using scRNA-seq. Nucleic Acids Res. (2020). doi:10.1093/nar/gkaa740

619 26. Rahmani, E. et al. Cell-type-specific resolution epigenetics without the need for cell sorting or single-cell biology. doi:10.1038/s41467-019-11052-9

27. Sassone-Corsi, P. Unique chromatin remodeling and transcriptional regulation in spermatogenesis. Science (2002). doi:10.1126/science.1070963

28. Daley, T. \& Smith, A. D. Predicting the molecular complexity of sequencing libraries. Nat. Methods (2013). doi:10.1038/nmeth.2375

29. Joo, M. S., Koo, J. H., Kim, T. H., Kim, Y. S. \& Kim, S. G. LRH1-driven transcription factor circuitry for hepatocyte identity: Super-enhancer cistromic analysis. EBioMedicine (2019). doi:10.1016/j.ebiom.2018.12.056 pathway substrate 8 (Eps8) is a novel regulator of cell adhesion and the blood-testis barrier integrity in the seminiferous epithelium. FASEB J. (2009). doi:10.1096/fj.06-070573

31. Liu, C. et al. An ATAC-seq atlas of chromatin accessibility in mouse tissues. Sci. Data (2019). doi:10.1038/s41597-019-0071-0 complex. Mol. Cell (2002). doi:10.1016/S1097-2765(02)00471-9 Commun. (2018). doi:10.1038/s41467-018-07270-2 
35. Whyte, W. A. et al. Master transcription factors and mediator establish super-enhancers at key cell identity genes. Cell (2013). doi:10.1016/j.cell.2013.03.035

36. Watt, A. J., Garrison, W. D. \& Duncan, S. A. HNF4: A central regulator of hepatocyte differentiation and function. Hepatology (2003). doi:10.1053/jhep.2003.50273

37. Stevens, J. et al. Analysis of the asymmetrically expressed Ablim1 locus reveals existence of a lateral plate Nodal-independent left sided signal and an early, left-right independent role for nodal flow. BMC Dev. Biol. (2010). doi:10.1186/1471-213X-10-54

38. Blendy, J. A., Kaestner, K. H., Weinbauer, G. F., Nieschlag, E. \& Schütz, G. Severe impairment of spermatogenesis in mice lacking the CREM gene. Nature (1996).

39. DeLaForest, A. et al. HNF4A is essential for specification of hepatic progenitors from human pluripotent stem cells. Development (2011). doi:10.1242/dev.062547

40. Subramanian, A. et al. Gene set enrichment analysis: A knowledge-based approach for interpreting genome-wide expression profiles. Proc. Natl. Acad. Sci. U. S. A. (2005). doi:10.1073/pnas.0506580102

41. Liu, Y., Zhou, J. \& White, K. P. RNA-seq differential expression studies: More sequence or more replication? Bioinformatics (2014). doi:10.1093/bioinformatics/btt688

42. Van den Berge, K. et al. RNA Sequencing Data: Hitchhiker's Guide to Expression Analysis. Annu. Rev. Biomed. Data Sci. 2, 139-173 (2019).

43. Sun, H. et al. Genome-wide mapping of RNA Pol-Il promoter usage in mouse tissues by ChIP-seq. Nucleic Acids Res. (2011). doi:10.1093/nar/gkq775

44. Tidball, J. G. Inflammatory processes in muscle injury and repair. American Journal of Physiology - Regulatory Integrative and Comparative Physiology (2005). doi:10.1152/ajpregu.00454.2004

45. Zeitlinger, J. et al. RNA polymerase stalling at developmental control genes in the Drosophila melanogaster embryo. Nat. Genet. (2007). doi:10.1038/ng.2007.26

46. Muse, G. W. et al. RNA polymerase is poised for activation across the genome. Nat. Genet. (2007). doi:10.1038/ng.2007.21 
48. De Micheli, A. J. et al. Single-cell analysis of the muscle stem cell hierarchy identifies heterotypic communication signals involved in skeletal muscle regeneration. doi:10.1101/671032

49. Dell'Orso, S. et al. Single cell analysis of adult mouse skeletal muscle stem cells in homeostatic and regenerative conditions. Dev. (2019). doi:10.1242/dev.174177

50. Oprescu, S. N., Yue, F., Qiu, J., Brito, L. F. \& Kuang, S. Temporal Dynamics and Heterogeneity of Cell Populations during Skeletal Muscle Regeneration. iScience (2020).

51. Naito, A. T. et al. Complement C1q activates canonical Wnt signaling and promotes aging-related phenotypes. Cell (2012). doi:10.1016/j.cell.2012.03.047

52. Wang, J. hua et al. Heterogeneous activation of a slow myosin gene in proliferating myoblasts and differentiated single myofibers. Dev. Biol. (2015).

53. Wosczyna, M. N. \& Rando, T. A. A Muscle Stem Cell Support Group: Coordinated Cellular Responses in Muscle Regeneration. Developmental Cell (2018). doi:10.1016/j.devcel.2018.06.018

54. Latroche, C. et al. Coupling between Myogenesis and Angiogenesis during Skeletal Muscle Regeneration Is Stimulated by Restorative Macrophages. Stem Cell Reports (2017). doi:10.1016/j.stemcr.2017.10.027

55. Core, L. J., Waterfall, J. J. \& Lis, J. T. Nascent RNA sequencing reveals widespread pausing and divergent initiation at human promoters. Science (80-. ). (2008). doi:10.1126/science.1162228

56. Rahl, P. B. et al. C-Myc regulates transcriptional pause release. Cell (2010).

57. Rahmani, E. et al. Cell-type-specific resolution epigenetics without the need for cell sorting or single-cell biology. Nat. Commun. (2019). doi:10.1038/s41467-019-11052-9 cellular mixtures based on linearity of transcriptional signatures. Nat. Commun. 10, 2209 (2019). 
699

700

701

702

703

704

705

706

707

708

709

710

711

712

713

714

715

716

717

718

719

720

721

722

68. Maehara, K. \& Ohkawa, Y. Agplus: A rapid and flexible tool for aggregation plots.

59. Ono, Y. et al. BMP signalling permits population expansion by preventing premature myogenic differentiation in muscle satellite cells. Cell Death Differ. (2011). doi:10.1038/cdd.2010.95

60. Kimura, H., Hayashi-Takanaka, Y., Goto, Y., Takizawa, N. \& Nozaki, N. The organization of histone $\mathrm{H} 3$ modifications as revealed by a panel of specific monoclonal antibodies. Cell Struct. Funct. (2008). doi:10.1247/csf.07035

61. Odawara, J. et al. The classification of mRNA expression levels by the phosphorylation state of RNAPII CTD based on a combined genome-wide approach. BMC Genomics 12, (2011).

62. Langmead, B. \& Salzberg, S. L. Fast gapped-read alignment with Bowtie 2. Nat. Methods (2012). doi:10.1038/nmeth.1923

63. Ramírez, F. et al. deepTools2: a next generation web server for deep-sequencing data analysis. Nucleic Acids Res. (2016). doi:10.1093/nar/gkw257

64. Patro, R., Duggal, G., Love, M. I., Irizarry, R. A. \& Kingsford, C. Salmon provides fast and bias-aware quantification of transcript expression. Nat. Publ. Gr. 14, (2017).

65. Zhang, Y. et al. Model-based analysis of ChIP-Seq (MACS). Genome Biol. (2008). doi:10.1186/gb-2008-9-9-r137

66. Schep, A. N., Wu, B., Buenrostro, J. D. \& Greenleaf, W. J. chromVAR: inferring transcription-factor-associated accessibility from single-cell epigenomic data. Nat. Methods 14, 975-978 (2017).

67. Heinz, S. et al. Simple Combinations of Lineage-Determining Transcription Factors Prime cis-Regulatory Elements Required for Macrophage and B Cell Identities. Mol. Cell (2010). doi:10.1016/j.molcel.2010.05.004

Bioinformatics (2015). doi:10.1093/bioinformatics/btv322 
bioRxiv preprint doi: https://doi.org/10.1101/2020 12 18.423434; this version posted December 18,2020 . The copyright holder for this

\section{Figure 1_Maehara}
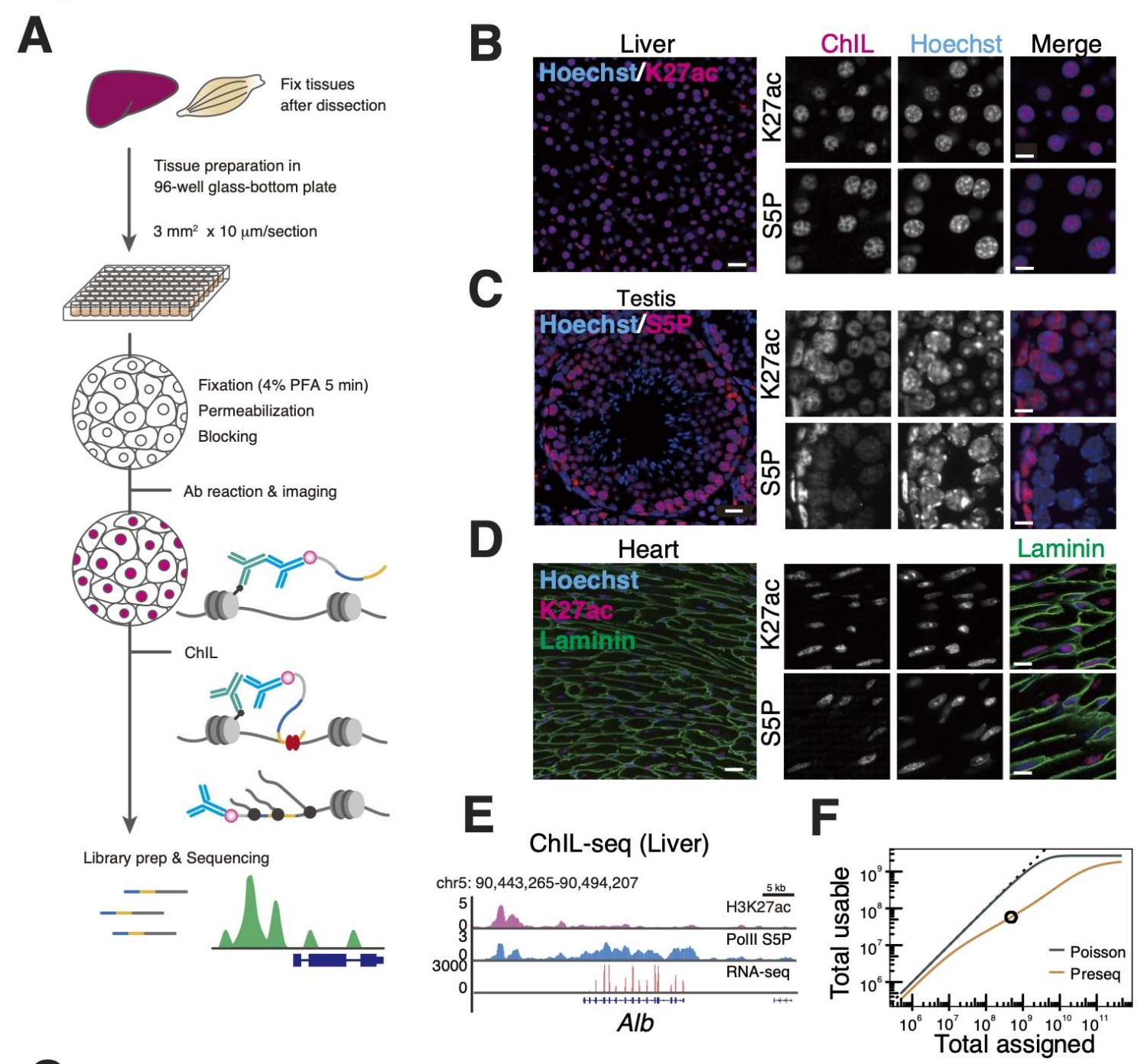

G

Library prep \& Sequencing
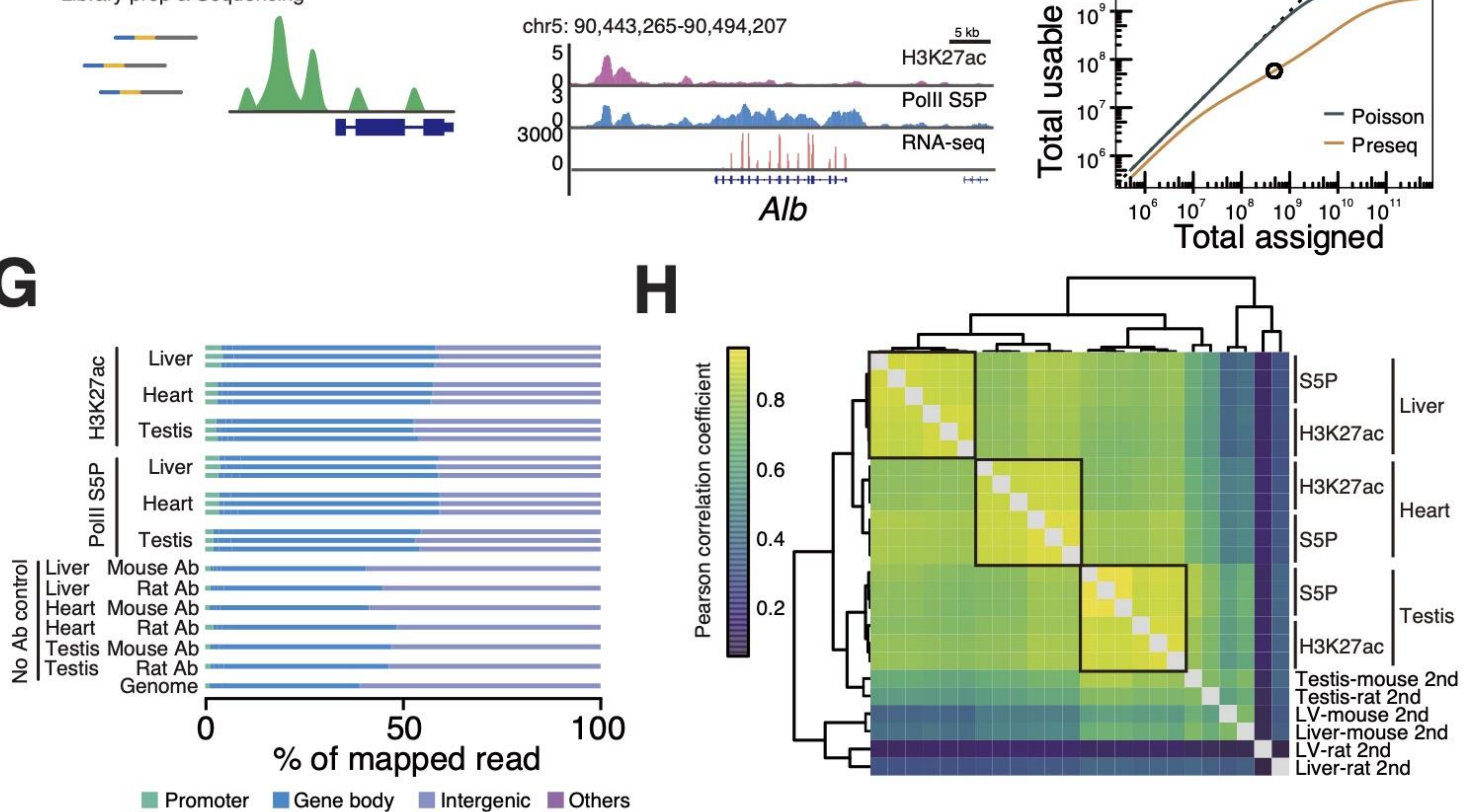

727 Figure 1: Epigenomic profiling using a single tissue section. (A) Schematic diagram of the

728 tsChIL protocol. (B-D) Immunofluorescent images of mouse liver (B), testis (C) and heart (D). 
729 H3K27ac, RNAPII-S5P, and laminin were stained with specific primary antibodies and visualized

730 using fluorescent labeled anti-mouse ChIL-probe (red: H3K27ac and PollIS5P) and anti-rabbit

731 IgG (green: laminin). DNA was counterstained with Hoechst 33342. Scale Bar: $20 \mu \mathrm{m}$ (left

732 images), $10 \mu \mathrm{m}$ (right images). (E) Genome browser images of ChIL-seq for H3K27ac and

733 RNAPII-S5P and bulk tissue RNA-seq data at the Alb locus in liver tissues. (F) Library complexity

734 of ChIL data. Poisson represents an ideal case of the uniform probability of obtaining reads from

735 the mouse genome, whereas preseq refers to the future/past predictions of a species discovery

736 curve of sequenced reads using Preseq ${ }^{28}$. Black circle indicates the read number we sequenced

737 for this prediction. (G) Breakdown of mapped reads at the annotated genomic regions. Gene

738 body: 3'-UTR, exon, intron, 5'-UTR; Others: ncRNA, miRNA, snoRNA, and pseudogenes. The

739 proportions of the annotated region on the mouse genome are shown as "Genome" at the bottom

740 lane. (H) Genome-wide correlation at $10 \mathrm{kbp}$ bins. Hierarchical clustering of Pearson's

741 correlation coefficient of log-transformed tsChIL-seq counts is shown. 


\section{Figure 2_Maehara}

A

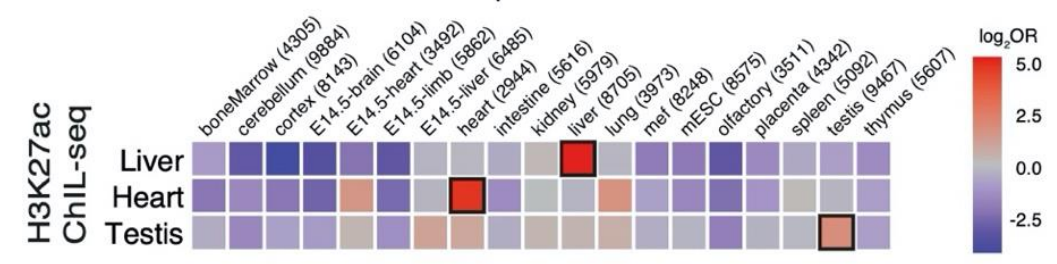

C

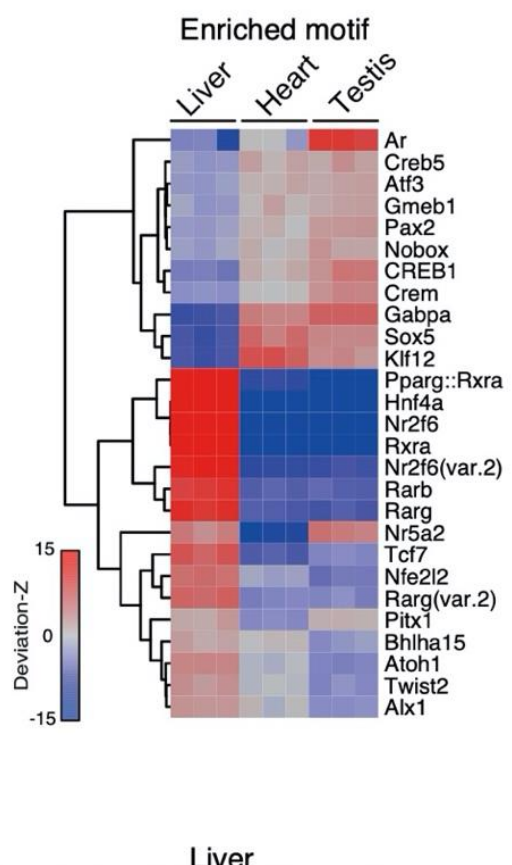

B

Chr2

Chr5 Chr6 Chr5

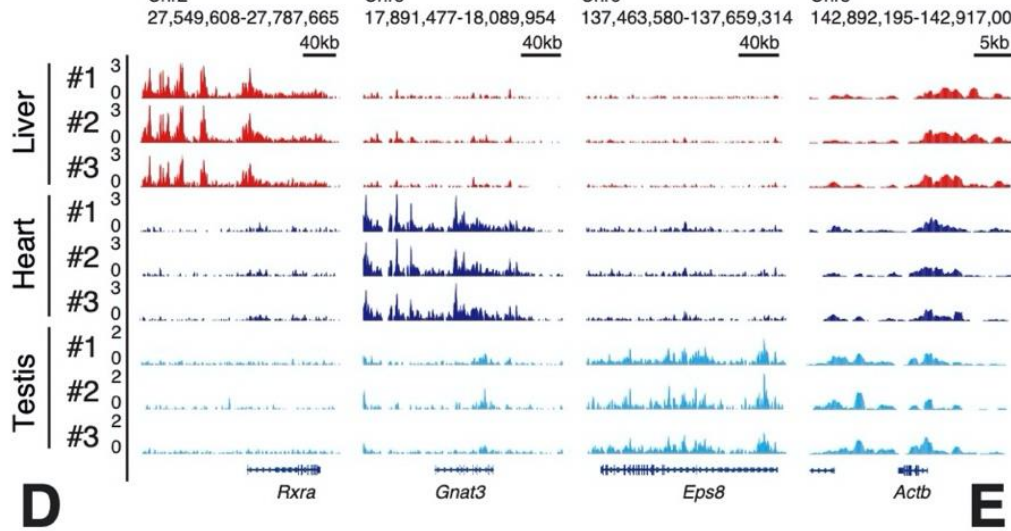

$5 \mathrm{~kb}$

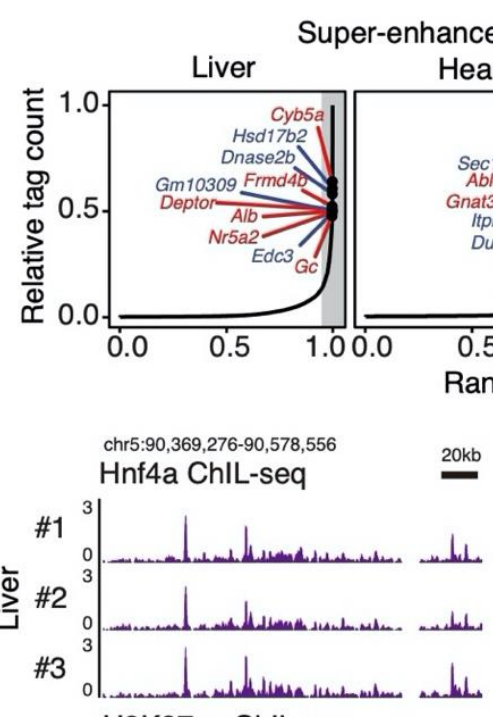

ancer analysis (a)
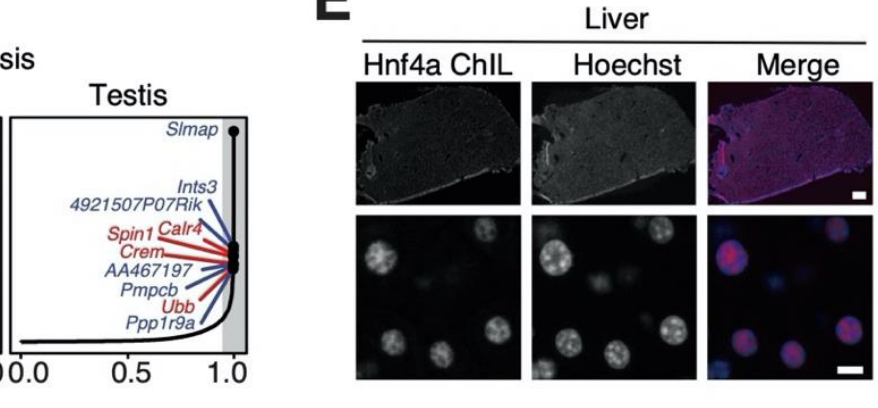

$\mathbf{F}$ H3K27ac ChlL-seq

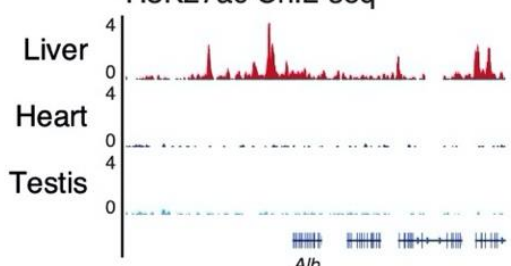

G
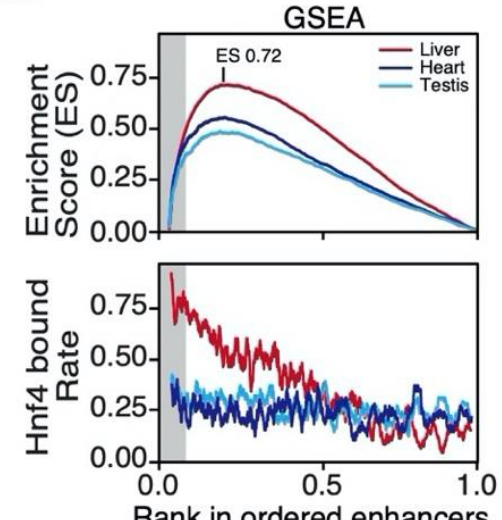

Alb

744 Figure 2: Upstream factor identification through enhancer analysis using tsChIL-seq. (A)

745 Tissue specificity of identified enhancers by tsChIL-H3K27ac. The odds ratios of hits in the reference tissue-specific enhancer list identified by bulk-tissue ChIP-seq data ${ }^{3}$ are shown. Odds 
747 is defined in Methods. The cells enclosed by black squares indicate the maximal odds ratios (i.e.,

748 maximal specificity) for each row. (B) The IGV tracks of tsChIL-H3K27ac at identified

749 tissue-specific enhancers of Rxra, Gnat3, Eps8, and a house-keeping gene of Actb loci are

750 shown with the replicates. (C) Specific motif enrichment analysis was conducted using

751 chromVAR ${ }^{66}$. Hierarchical clustering of deviation-Z scores of three replicates of each tissue is

752 shown. (D) Super-enhancer identification. Tissue-specific enhancers are identified, so that they

753 are listed more than twice (twice: blue, all: red) in the top 5\% in all enhancer candidates and are

754 not in the SEs of other tissues. Grey shades indicate the top $5 \%$ of tag count in enhancer

755 candidates. (E) Immunofluorescent images of mouse liver sections. Tissues were stained with

756 anti-Hnf4a antibody and visualized by a fluorescent-labeled anti-mouse ChIL probe. DNA was

757 counterstained with Hoechst 33342. Scale bar: $200 \mu \mathrm{m}$ (top), $10 \mu \mathrm{m}$ (bottom). (F) Hnf4a binds to

758 the SE at Alb gene loci. (G) Gene set enrichment analysis of Hnf4a-bound genes (top), and their

759 rate of Hnf4a-bound genes in sliding windows of 100 genes (bottom). 


\section{Figure 3_Maehara}

A

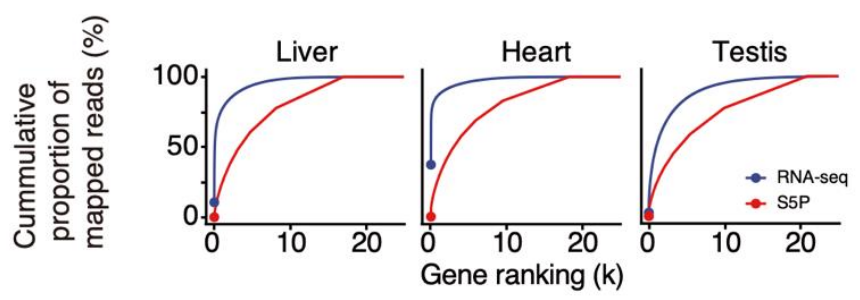

B

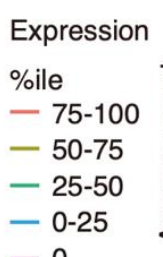

$-0$

E

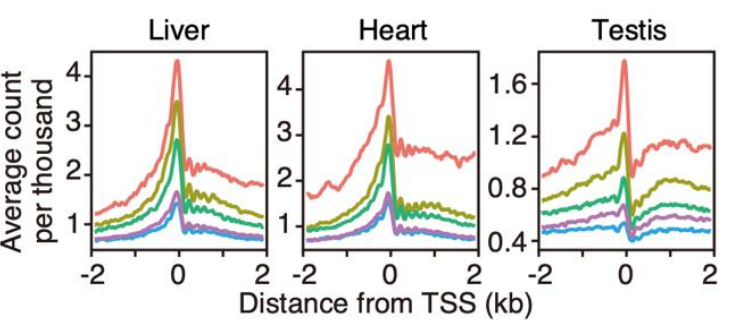

D
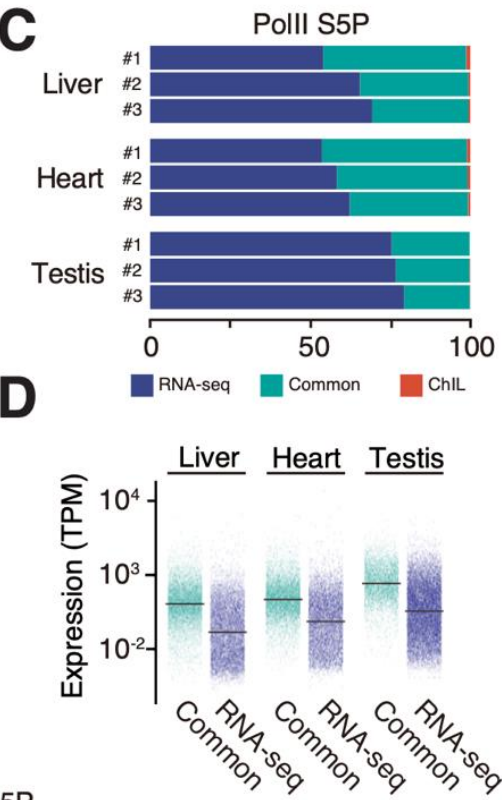

Tissue-specific peaks of Polll S5P
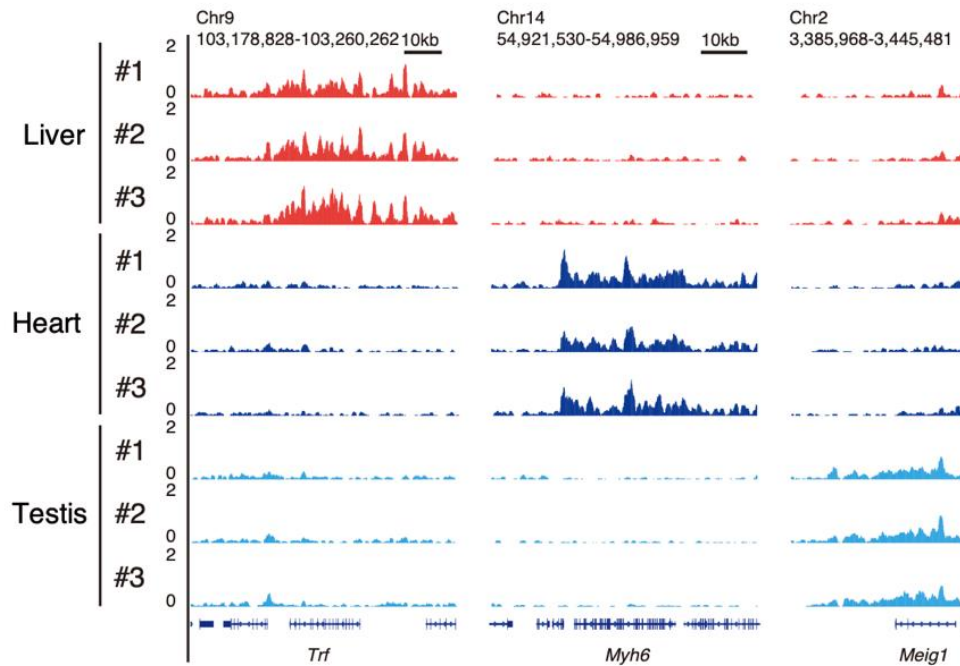

$10 \mathrm{~kb} \quad 142$

$142,880,311-142,929,558 \quad 10 \mathrm{~kb}$
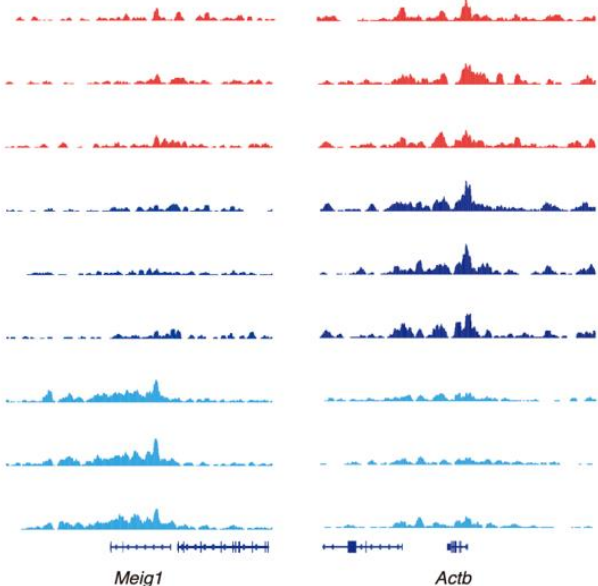

Figure 3: ChIL-RNAPII detect active genes in tissue. (A) Dynamic ranges of bulk-tissue

RNA-seq and tsChIL RNAPII. The cumulative proportion in total mapped reads at genes (red: tsChIL, blue: RNA-seq) were compared. Genes are ordered by the read counts on the exons for RNA-seq and on +/-750 bp from TSS for tsChIL, respectively. (B) Signal intensities of tsChIL correlated with the expression levels of genes. The lines indicate the average CPM of each expression group at TSS. The expression groups were assigned with respect to the expression levels (TPM) of genes. (C) Coverage of expressed genes by tsChIL-RNAPII peaks. The stacked bar chart shows the proportions of detected genes in the RNA-seq only (RNA-seq: blue), tsChIL-Pol2 only (ChIL: red) and both (Common: green). (D) Higher expression levels at tsChIL-Pol2 peaks. The expression levels of all expressed genes (TPM $>0$ ) are shown. (E) The 
bioRxiv preprint doi: https://doi.org/10.1101/2020.12.18.423434; this version posted December 18, 2020. The copyright holder for this preprint (which was not certified by peer review) is the author/funder, who has granted bioRxiv a license to display the preprint in perpetuity. It is made available under aCC-BY 4.0 International license.

772 tissue-specific genes identified by tsChIL-RNAPII. The IGV tracks of all replicates of

773 tsChIL-RNAPII are shown at each specific gene (Trf, Myh6, and Meig for the liver, heart, and

774 testis, respectively). Actb is also shown as the ubiquitously expressed gene in the three tissues. 
Figure 4_Maehara

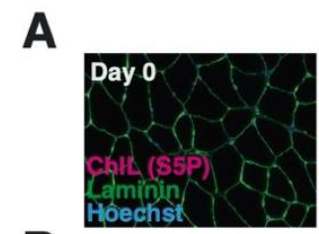

B

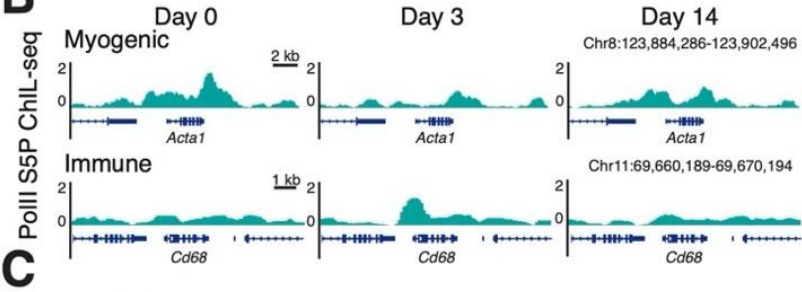

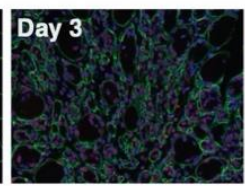

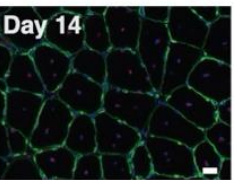

Day 14
E

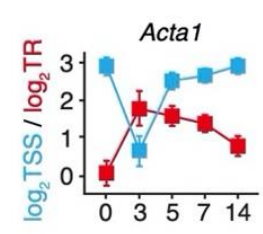

D

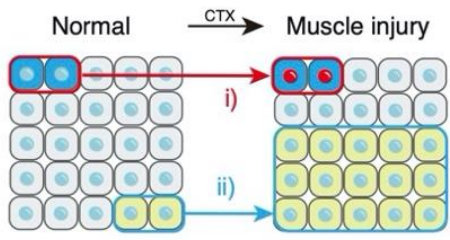

i) Transcriptional activation
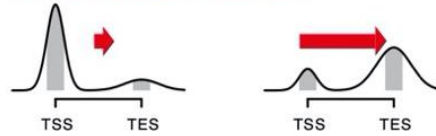

ii) Population size increment
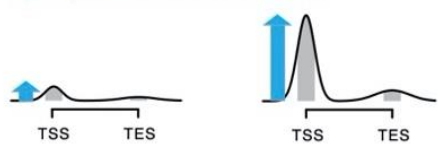

F
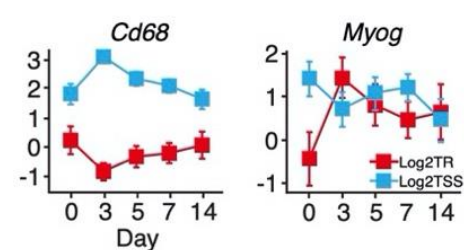

G

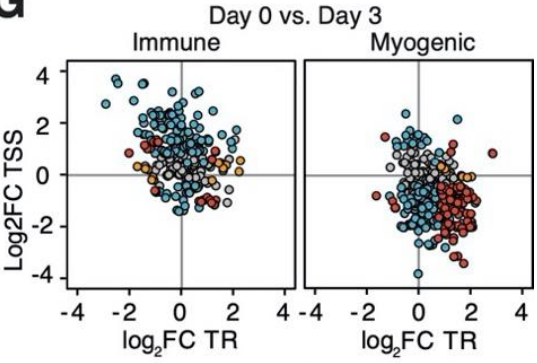

FDR $<0.1$ at oNone oTSS OTR oBoth

H

$\log _{2}$ TR $\log _{2}$ TSS

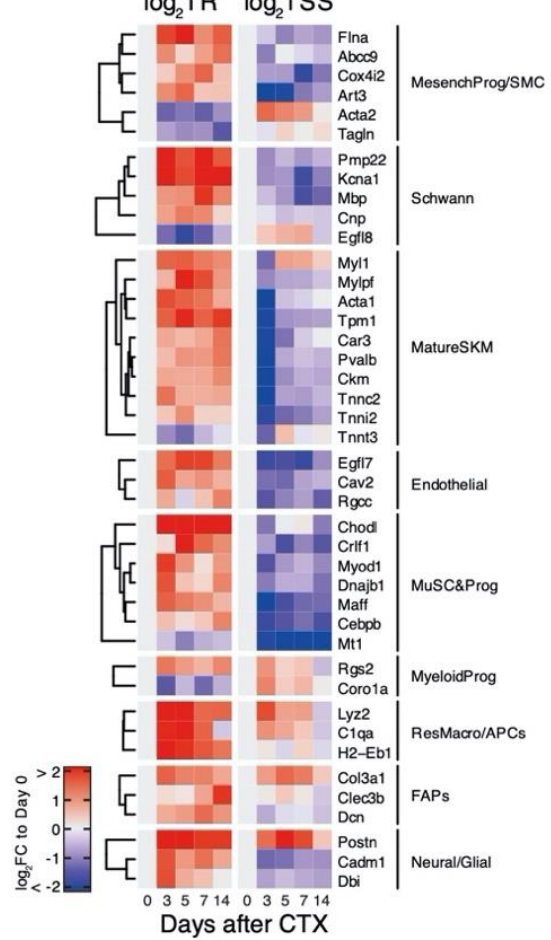

I

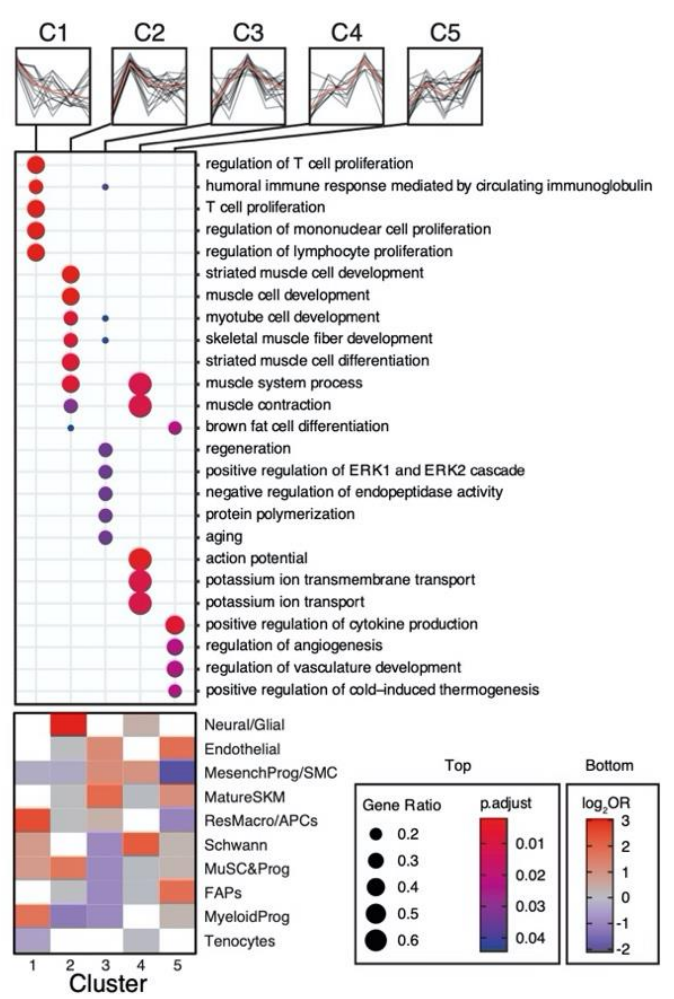

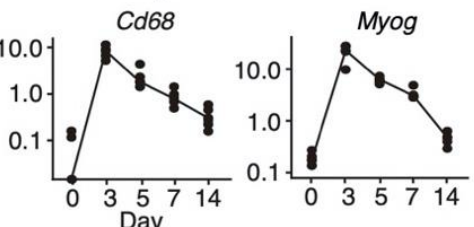


776 Figure 4: Statistical modeling of the traveling ratio reveals the independent dynamics

777 between population and transcriptional regulation in regenerating skeletal muscle

778 tissues. (A) Immunofluorescent images of the mouse tibialis anterior muscle on the indicated

779 days after CTX treatment. The images of anti-mouse ChIL probe for RNAPII-S5P (red) and

780 anti-rabbit lgG for laminin (green) are shown. Scale bar: $20 \mu \mathrm{m}$. Refer to Figure S6A for more

781 frequent time points. (B) tsChIL-RNAPII signal of the marker genes of mature skeletal muscle

782 (Acta1) and macrophages (Cd68). (C) Proportion of sequenced reads (\%UMI) occupied by the

783 representative cell types in muscle regeneration. The single cell data (GSE143437) by De

784 Micheli et al. ${ }^{48}$ was re-analyzed. See Figure S6B for the detailed cell-type annotations. (D)

785 Extraction of independent dynamics of the population and transcriptional regulation. Change in

786 RNAPII distribution at the gene loci: a gene (blue) was transcriptionally activated (red nuclei)

787 following the stimuli, while population size was unchanged. Change in the height of RNAPII

788 distribution: a type of cells (yellow) was grown after the stimuli, while the transcriptional activity

789 was maintained. (E) Estimated mean (95\% confidence interval) of TR and the CPM of

790 tsChIL-RNAPII at TSS. Representative genes of mature skeletal muscle cells and immune cells

791 are shown. (F) Bulk-tissue expression levels (TPM) of the representative genes. (G) Different

792 activities of two major cell-types in muscle regeneration. Scatter plots of $\log _{2} \mathrm{FC}$ of day-3 vs.

793 day-0 of TR (x-axis) and the TSS-level (y-axis) are shown: immune cell marker genes (left);

794 myogenic genes: right. Colors indicate significance in TR and TSS-level based on $\left|\log _{2} \mathrm{FC}\right|>1$

795 (two-fold) and FDR < 0.1. (H) Activities of major cell types in muscle regeneration. The colors of

796 the heatmap show the $\log _{2} \mathrm{FC}$ to day 0 (uninjured) of TR and TSS levels. Representative genes

797 with significant changes in TR are shown. (I) The dynamics of the biological process in muscle

798 regeneration and the participating cell types. Genes were assigned to five groups (C1-5) based

799 on highest time point of TR. OR indicates the specificity of participation to the biological

800 processes.

801 Table 1: Cell numbers in the tissue sections used in this study

\begin{tabular}{|c|c|c|c|c|}
\hline \multirow{2}{*}{$\begin{array}{l}\text { Tissue section } \\
\text { Heart H3K27ac }\end{array}$} & \multicolumn{3}{|c|}{ Cell count (rep.\#1-3) } & \multirow{2}{*}{$\begin{array}{r}\text { Average } \\
11,866\end{array}$} \\
\hline & 12,297 & 14,210 & 9,090 & \\
\hline Heart RNAPII-S5P & 12,155 & 13,755 & 8,847 & 11,586 \\
\hline Liver H3K27ac & 14,999 & 7,458 & 11,551 & 11,336 \\
\hline Liver RNAPII-S5P & 14,177 & 14,112 & 16,330 & 14,873 \\
\hline Testis H3K27ac & 17,085 & 16,723 & 16,931 & 16,913 \\
\hline Testis RNAPII-S5P & 20,542 & 17,426 & 13,634 & 17,201 \\
\hline TA muscle RNAPII-S5P & 4,104 & 3,723 & 4,421 & 4,083 \\
\hline
\end{tabular}

802 
bioRxiv preprint doi: https://doi.org/10.1101/2020.12 18.423434; this version posted December 18,2020 . The copyright holder for this preprint (which was not certified by peer review) is the author/funder, who has granted bioRxiv a license to display the preprint in perpetuity. It is made available under aCC-BY 4.0 International license.

\section{Supplementary Information}
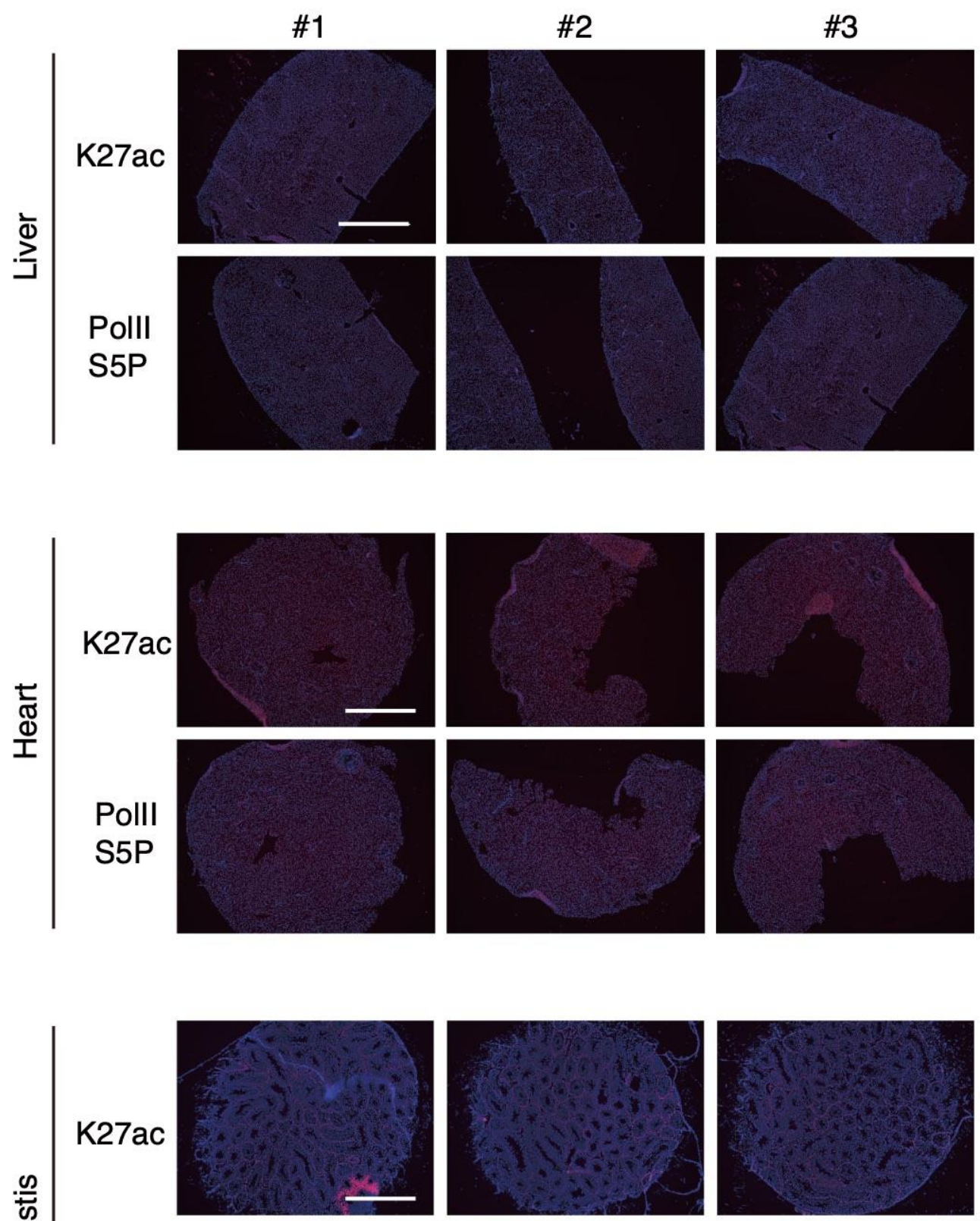

$\stackrel{\oplus}{\oplus}$
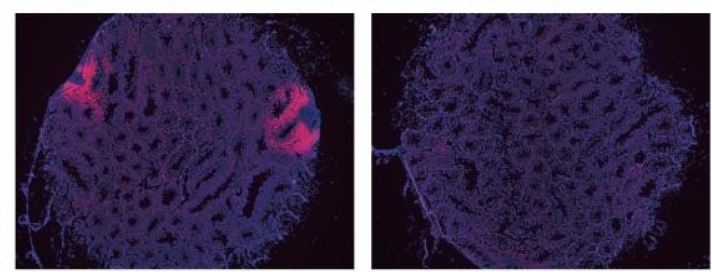

Figure S1: Immunofluorescent images of whole sections stained with the ChIL-probe. Immunofluorescent images of the indicated tissues for all replicates $(N=3)$. Tissue sections were stained with H3K27ac or PollIS5P antibody and visualized using the fluorescent dye-conjugated ChIL-probe. DNA was counterstained with Hoechst 33342. Scale bar: $1 \mathrm{~mm}$. 
Super-Enhancer analysis

Liver
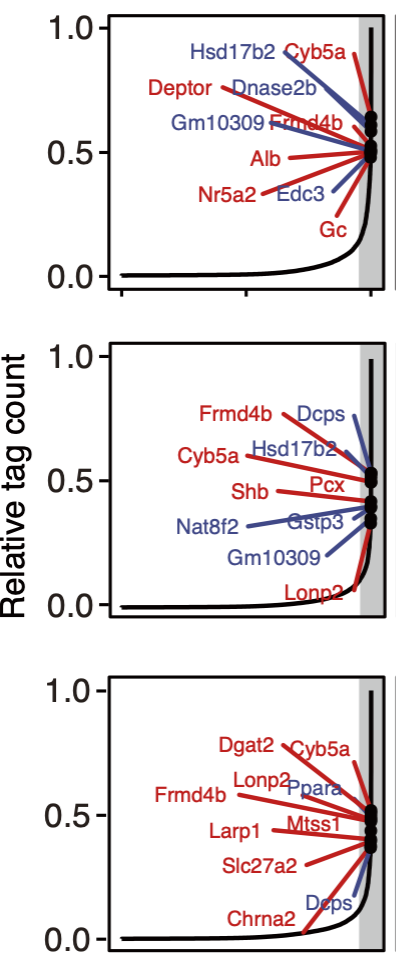

Heart
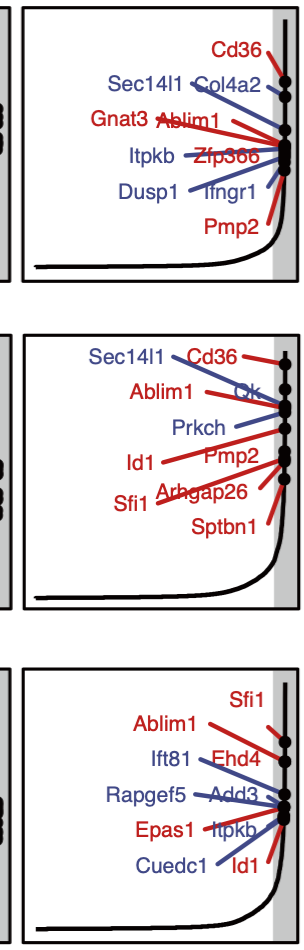

Testis

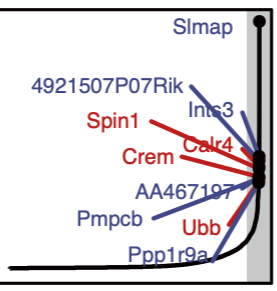

Rep.1

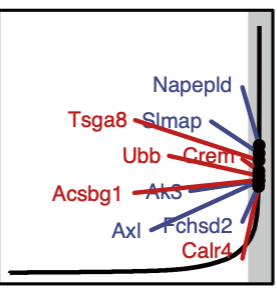

Rep.2

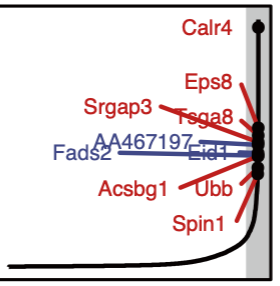

Rep.3

810 Figure S2: Super-enhancer analysis of each replicate. Tissue-specific enhancers are identified so that they are listed more than twice (twice: blue, all: red) in the top 5\% of tag count among enhancer candidates and are not in the SEs of other tissues. Grey shades indicate the top $5 \%$ of tag count among the enhancer candidates.

CAGAGGTCAAATCCA HNF4a(NR),DR1/HepG2-HNF4a-ChIP-Seq(GSE25021)/Homer $\quad-1432$

Figure S3: Motif enrichment analysis of tsChIL-Hnf4a peaks. Enrichment analysis of known enrichment of motifs was evaluated within $250 \mathrm{bp}$ from a summit of MACS2 peaks. The height of the motif logos corresponds to nucleotide frequencies. 
A

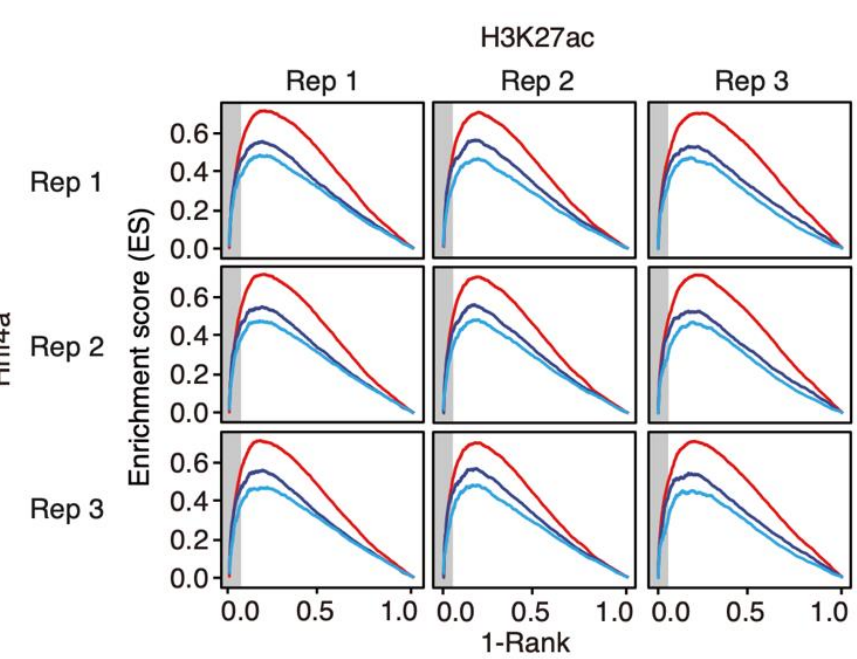

B

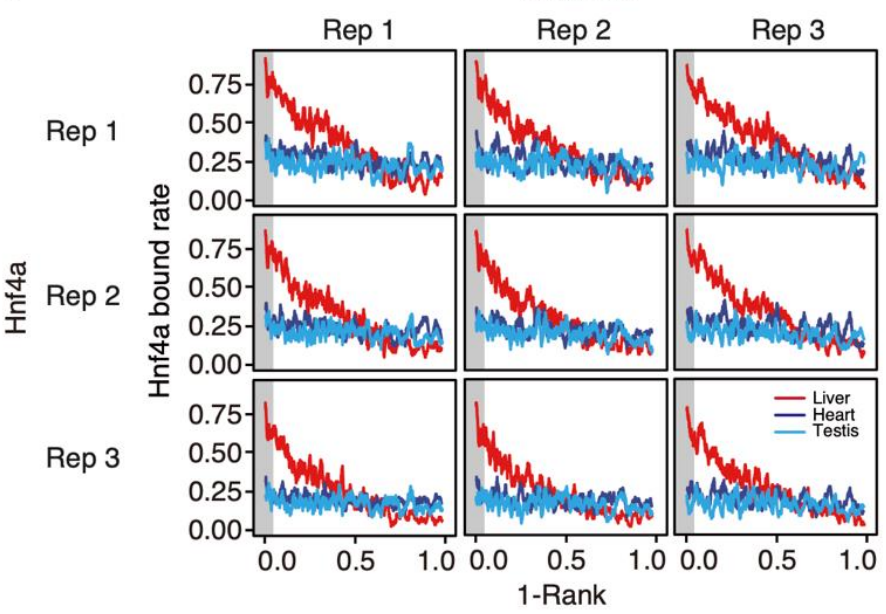

822 Figure S4: Enhancer set enrichment analysis of Hnf4a-bound genes. Gene set enrichment analysis of Hnf4a-bound genes (A), and the rate of Hnf4a-bound genes in the sliding windows of 100 genes $(B)$ along the ordered enhancers. All possible combinations $(3 \times 3$ combination of replicates for tsChIL-H3K27ac and tsChIL-Hnf4a) are shown.
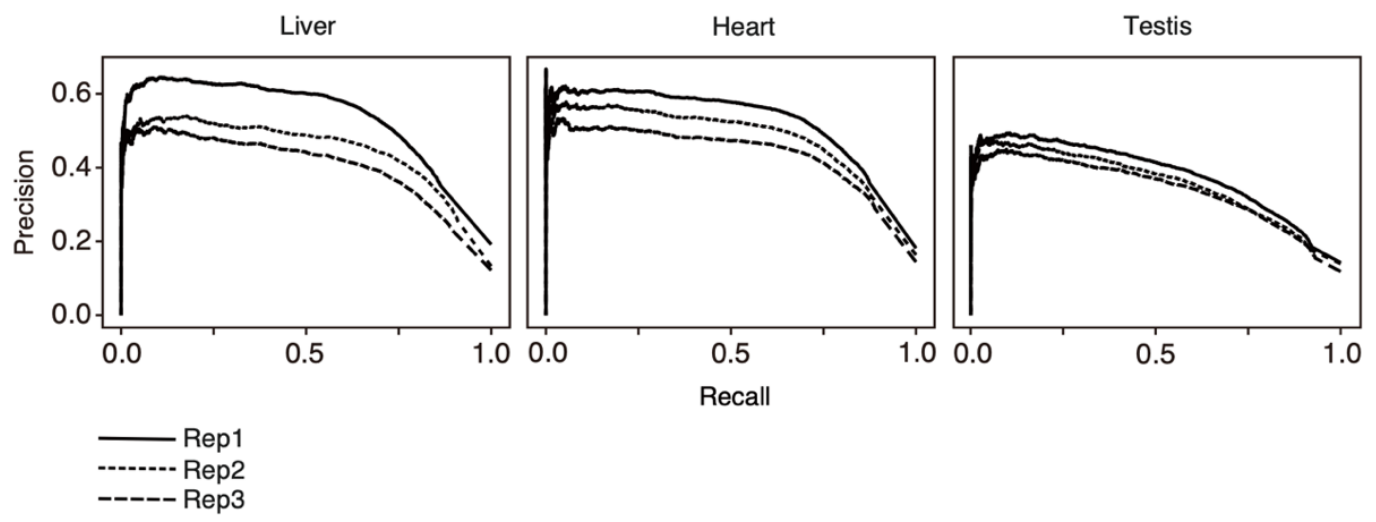

Figure S5: Tolerant definition of "active genes" by RNA-seq. Precision and recall curves for predicting tsChIL-RNAPII peaks based on TPM values are shown. The recall represents the proportion of RNAPII peaks covered by the active genes, and the precision is the proportion of active genes covered by the RNAPII peaks. Active genes are defined at each TPM threshold. 
A
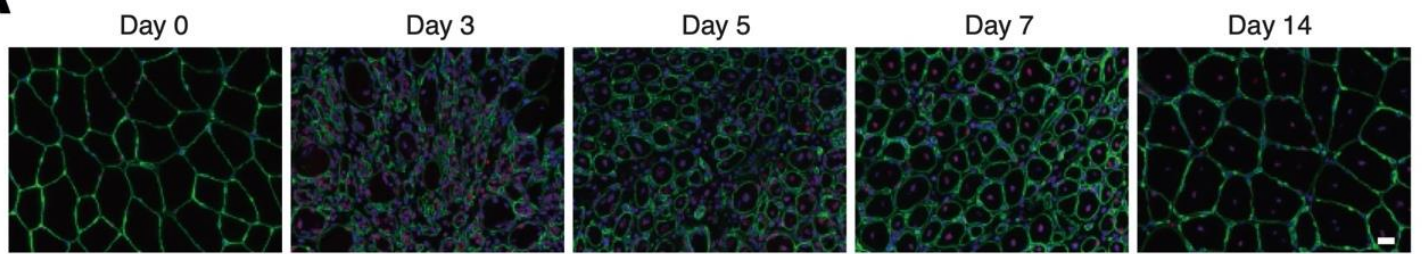

B

ChIL (S5P) Laminin Hoechst

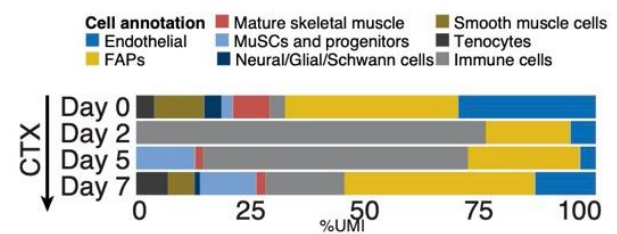

C
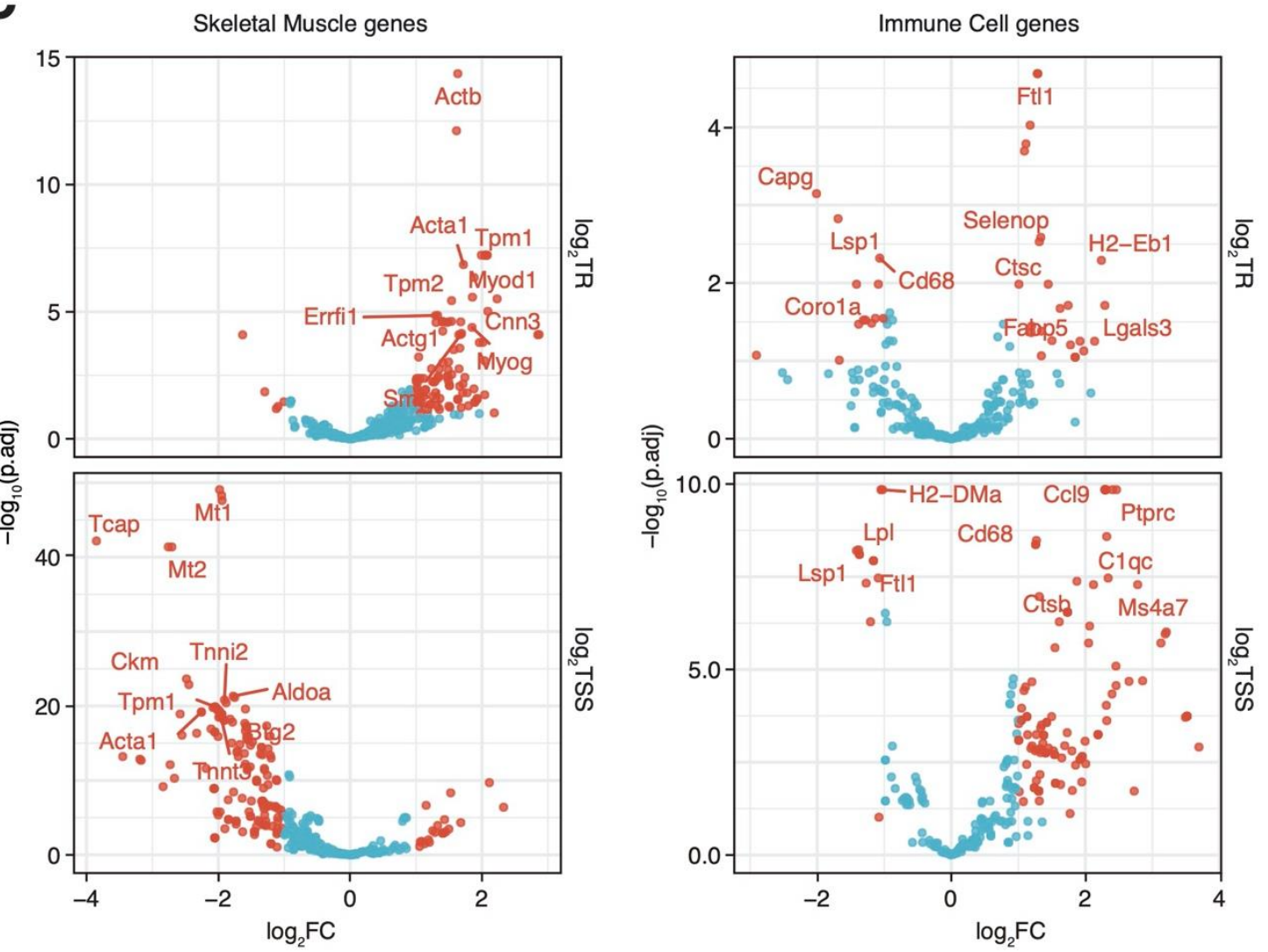

Figure S6: Statistical modeling of RNAPII reveals transcriptional dynamics in muscle regeneration. (A) The complete immunofluorescent images shown in Figure 4a. Scale bar: 20 $\mu \mathrm{m}$. (B) Proportion of sequenced reads (\%UMI) occupied by the annotated cell types in muscle regeneration. The single cell data (GSE143437) by De Micheli et al. was re-analyzed. (C) Volcano plots of the contrasts (day 3 vs. day 0 after CTX injury) for TR (top) and TSS (bottom). The $x$-axis represents $\log _{2} F C$ (day $3 /$ day 0 ), whereas the $y$-axis represents $-\log _{10} F D R$. Significant changes that satisfy $\left|\log _{2} \mathrm{FC}\right|>1$ (twofold) and FDR $<0.1$ are in red. Genes that have the top $10 p$-values are labelled. 
bioRxiv preprint doi: https://doi.org/10.1101/2020.12.18.423434: this version posted December 18,2020 . The copyright holder for this preprint (which was not certified by peer review) is the author/funder, who has granted bioRxiv a license to display the preprint in perpetuity. It is made available under aCC-BY 4.0 International license.

841 Table S1: Epigenomic analysis methods on tissue sections

842

\begin{tabular}{|c|c|c|c|c|}
\hline Method & Tissue & Target & Sample vol. & Journal \\
\hline PAT-ChIP & Spleen & Histone modification & 4 sections & $\begin{array}{l}\text { Fanelli et al., } 2010 \\
\text { Proc Natl Acad Sci USA } \\
\text { Fanelli et al., } 2011 \\
\text { Nat Protoc }\end{array}$ \\
\hline FiT-seq & $\begin{array}{l}\text { Seminoma } \\
\text { Breast cancer } \\
\text { Bladder cancer } \\
\text { CRC }\end{array}$ & Histone modification & 10 sections & $\begin{array}{l}\text { Cejas et al., } 2016 \\
\text { Nat Med }\end{array}$ \\
\hline EPAT-ChIP & Breast cancer & Histone modification & 10 sections & $\begin{array}{l}\text { Amatori et al., } 2018 \\
\text { Clin Epigenetics }\end{array}$ \\
\hline $\begin{array}{l}\text { Chrom-EX } \\
\text { PE }\end{array}$ & $\begin{array}{l}\text { Liver } \\
\text { Spleen }\end{array}$ & $\begin{array}{l}\text { Histone modification } \\
\text { Polymerase }\end{array}$ & 2 sections & $\begin{array}{l}\text { Zhong et al., } 2019 \\
\text { BMC Genomics }\end{array}$ \\
\hline FiTAc-seq & $\begin{array}{l}\text { Seminoma } \\
\text { Breast cancer } \\
\text { Bladder cancer } \\
\text { Melanoma } \\
\text { PNETs }\end{array}$ & Histone modification & 2-4 sections & $\begin{array}{l}\text { Font-Tello et al., } 2020 \\
\text { Nat Protoc }\end{array}$ \\
\hline $\begin{array}{l}\text { Tissue-ChIL } \\
\text {-seq }\end{array}$ & $\begin{array}{l}\text { Liver } \\
\text { Heart } \\
\text { Testis } \\
\text { Skeletal muscle }\end{array}$ & $\begin{array}{l}\text { Histone modification } \\
\text { Transcription factor } \\
\text { Polymerase }\end{array}$ & 1 section & This study \\
\hline
\end{tabular}

